\title{
In-depth Insights into Alzheimer's Disease by using Explainable Machine Learning Approach - Existing Hypothesis Confronted
}

Bojan Bogdanovic ( $\sim$ bojanbogdanovic96@gmail.com )

UKIM: Saints Cyril and Methodius University in Skopje https://orcid.org/0000-0001-9248-3406

Tome Eftimov

Institut Jožef Stefan: Institut Jozef Stefan

Monika Simjanoska

UKIM: Saints Cyril and Methodius University in Skopje

\section{Research}

Keywords: Alzheimer's disease, Machine Learning, Explainability, Shapley values, Global interpretability, Local interpretability. Hypothesis testing

Posted Date: May 5th, 2021

DOl: https://doi.org/10.21203/rs.3.rs-447958/v1

License: (c) (i) This work is licensed under a Creative Commons Attribution 4.0 International License. Read Full License 


\title{
In-depth Insights into Alzheimer's Disease by using Explainable Machine Learning Approach - Existing Hypothesis Confronted
}

\author{
Bojan Bogdanovic ${ }^{1 *}$, Tome Eftimov ${ }^{2}$ and Monika Simjanoska ${ }^{1,3}$ \\ for the Alzheimer's Disease Neuroimaging Initiative ${ }^{4}$
}

${ }^{*}$ Correspondence: bo-

jan.bogdanovic@students.finki.ukim.mk

${ }^{1}$ Faculty of Computer Science and

Engineering, Ss. Cyril and

Methodius University, Rugjer

Boshkovikj 16, 1000 Skopje, N.

Macedonia

Full list of author information is

available at the end of the article

\begin{abstract}
Background: Alzheimer's disease is still a field of research with lots of open questions. The complexity of the disease prevents the early diagnosis before visible symptoms regarding the individual's cognitive capabilities occur. This research presents an in-depth analysis of a huge data set encompassing medical, cognitive and lifestyle's measurements from more than 12,000 individuals. Several hypothesis were established whose validity has been questioned considering the obtained results.
\end{abstract}

Methods: The importance of appropriate experimental design is highly stressed in the research. Thus, a sequence of methods for handling missing data, redundancy, data imbalance, and correlation analysis have been applied for appropriate preprocessing of the data set, and consequently Random Forest and XGBoost models have been trained and evaluated with special attention to the hyperparameters tuning. Both of the models were explained by using the Shapley values produced by the SHAP method.

Results: XGBoost produced the best f1-score of 0.84 and as such is considered to be highly competitive among those published in the literature. This achievement, however, was not the main contribution of this paper. This research's goal was to perform global and local interpretability of both the intelligent models and derive valuable conclusions over the established hypothesis. Those methods led to a single scheme which presents either positive, or, negative influence of the values of each of the features whose importance has been confirmed by means of Shapley values. This scheme might be considered as additional source of knowledge for the physicians and other experts whose concern is the exact diagnosis of early stage of Alzheimer's disease.

Conclusion: The conclusions derived from the intelligent models interpretability rejected all the established hypothesis. This research clearly showed the importance of Machine learning explainability approach that opens the black box and clearly unveils the relationships among the features and the diagnoses.

Keywords: Alzheimer's disease; Machine Learning; Explainability; Shapley values; Global interpretability; Local interpretability; Hypothesis testing

\section{Introduction}

Alzheimer's disease (AD) is considered to be common cause of dementia worldwide [1]. Over time, people with Alzheimer's disease suffer memory loss as well as the ability to concentrate. In advanced stages of the disease, there are severe compli- 
cations such as dehydration, malnutrition or infection, which eventually result in death [2]. Since its first description in the early twentieth century, there is still no treatment that cures Alzheimer's disease or alters the disease process in the brain. However, the course of Alzheimer's can be positively influenced by a number of different medications and non-drug treatments, making it crucial for people with Alzheimer's to receive early good care and support.

Even associated with forgetfulness, AD affects different aspects of individual's personality, life experiences, current circumstances and wrong responses to the situations they suddenly find themselves in [3], and relationships with other people as is the verbal communication. The gradual nature of affecting the short-term memory at first and the long-term memory at later stages makes the maintenance of orientation in time and space inevitably difficult. Figure 1 presents a time-series of self-portraits of an artist as his Alzheimer's disease progresses in time [4] and the original work clearly shows the cognitive decline and spatial disorientation, however, the emotion still highly remains in each of the original works.

It is not yet possible to diagnose Alzheimer's with complete certainty using the currently available tests while the person is still alive. The disease is diagnosed if someone has the typical symptoms eliminating all the other possible causes. Since symptoms like forgetfulness, changes in behavior and problems with orientation might have many different causes, it is important not to rush to a diagnosis of Alzheimer's. The symptoms might also be caused by depression or other physical conditions like meningitis, a stroke or bleeding in the brain [5]. Conducting an effective clinical trial is crucial to accurately predict the change in AD's indicators so that the effect of the treatment can be assessed. The most common approaches are:

- Manual prediction by a clinical expert by using the clinical history of patients with similar conditions and visual analysis of various brain scans.

- Regression analysis to predict the future indicator changes in patient status, based on data from MRI [6], cognitive test scores [7], rate of cognitive decline [8], and also retrospectively staging subjects by time to conversion between diagnoses [9].

- Supervised Machine Learning (ML) has already shown to be effective in discrimination between AD patients from cognitively normal subjects by using MR images [10], variety of biomarkers [11], etc.

- Data-driven disease progression models are most recently used to predict AD in unsupervised manner. Examples include models built on a set of biomarkers to produce discrete $[12,13]$ or continuous $[14,15]$ pictures of disease progression. Also there are less comprehensive models that leverage structure in data such as MR images [16, 17].

Even though it is still unclear what is the main cause of the disease, it has been shown that people with Alzheimer's do not have enough of an important chemical messenger called acetylcholine in their brain [18]. And it has also been shown that small protein particles (for example plaques) build up in their brain. These might cause the nerve cells to die [19].

In [20] several factors have been hypothesized to play a role in the Alzheimer's disease occurrence, and those are: 
- Age - starting at about age of 65 , the probability of getting AD doubles every 5 years $[21,22]$.

- apolipoprotein E4 (APOE E4) - 10 to 30 times higher of developing AD compared to non-carriers, i.e., subjects without the gene. However the exact mechanism through which the presence of APOE E4 leads to AD is not known [23].

- Gender - women seem more likely to develop AD than men. The reasons for this are still unclear [24].

- Medical conditions - type 2 diabetes, high blood pressure, high cholesterol, obesity [25], or depression [26] are known to increase the risk of developing dementia.

- Lifestyle factors - physical inactivity [27], smoking [28], unhealthy diet [29], excessive alcohol [30], or head injuries [31].

\subsection{Hypothesis}

This research is focused on deep investigation of some of the factors that are claimed to play an important role for the occurrence and further development of Alzheimer's disease by following explainable Machine Learning (ML) approach. The research puts the following hypothesis at test:

- Hypothesis 1: There is gender predisposition for obtaining Alzheimer's disease.

- Hypothesis 2: APOE4 gene is crucial decisive factor for Alzheimer's disease diagnosis.

- Hypothesis 3: Older people are more prone to Alzheimer's disease.

- Hypothesis 4: Cognitive tests distinguish between all stages leading to Alzheimer's disease.

To test the established hypothesis, a data set encompassing 12,741 subjects medically confirmed to belong into five categories - cognitively normal (CN), Early Mild Cognitive Impairment (EMCI), Late Mild Cognitive Impairment (LMCI), Significant Memory Concern (SMC) or Alzheimer's disease (AD), will be used to develop intelligent models able to classify the patients with high precision and recall with the aim to further interpret the models by the explainable ML methods.

The paper is organized as follows. The data set is fully described in Section 2. In the same Section 2 there is a comprehensive description of the data preprocessing as well as the ML approaches applied to the data set. The intelligent model's results are provided in Section 3. The models are interpreted by means of explainable ML in Section 2.5 upon which a discussion showing the importance of the research is provided in Section 4. Section 5 presents the final conclusion over the established hypotheses.

\section{Materials and methods}

Figure 2 presents the complete methodology as a roadmap followed to analyse and derive valuable conclusions from the data. Six different phases can be distinguished encompassing methods as follows:

- Phase 1: Data set analysis. In this step the subjects and all the features available in the database are analysed in terms of their importance for the problem at hand. 
- Phase 2: Data set preprocessing. This phase is focused on methods for incomplete data, redundant features, encoding, correlation analysis, missing values, and imbalanced targets handling, from which as a result a data set prepared for ML will be obtained.

- Phase 3: Training ML models. At the ML phase, Random Forest and XGBoost models have been trained, and also hyperparameters tuning has been performed.

- Phase 4: Explainability. This is the most important phase considering the contribution of the research, since it allows the global and local interpretability of the trained models by using Shapley values and thus, provides deep insight into the features influence on the prediction of each of the classes.

- Phase 5: Valuable conclusions. Based on the previous phase, valuable conclusions are derived at this phase followed by comprehensive discussion.

- Phase 6: Hypothesis testing. At the last phase, the research is concluded by reconsidering the established hypothesis based on the results from Phase 5.

\subsection{Data set analysis}

The data set used in this research (TADPOLE) is retrieved from ADNI (Alzheimer's Disease Neuroimaging Initiative) available at http://adni.loni.usc.edu/. In order to obtain permission for data usage, a request has been sent together with an explanation for the purpose of the research.

The original data set contains data from 12,741 subjects and 1,907 attributes. Mainly the attributes arise from two categories: quantitative parameters and personal information. As provided in the data set description, the data is labeled by medical experts.

Considering the sparse data set problem and according to the suggestions provided in the data set source [32], 17 features have been chosen to be informative for most of the patients covering personal information, gene expression analysis, medical information and cognitive tests information. Those feature are considered to carry information needed to test the hypothesis established in Section 1.1.

Each row represents data for one subject, and each column represents a feature or measurement related to the subject. The features can be divided into six categories, encompassing:

\section{- Personal information:}

- PTID: Participant ID

- AGE: Age at baseline

- PTGENDER: Sex

- PTEDUCAT: Years of education

- PTRACCAT: Race

\section{- Gene expression:}

- APOE4: Expression of the ApoE4 gene

\section{- PET measures:}

- FDG: measure cell metabolism, where cells affected by AD show reduced metabolism.

- AV45: measures amyloid-beta load in the brain, where amyloid-beta is a protein that mis-folds (i.e. its 3D structure is not properly constructed), which then leads to AD. 
- MRI measures:

- Hippocampus: scan of a complex brain structure embedded deep into temporal lobe.

- WholeBrain: scan of the subject's whole brain.

- Entorhinal: scan of an area of the brain that is located in the medial temporal lobe and functions as a hub in a widespread network for memory, navigation and the perception of time.

- MidTemp: scan of the middle temporal artery.

- Cognitive tests:

- CDRSB: Clinical Dementia Rating Scale - Sum of Boxes.

- ADAS11: Alzheimer's Disease Assessment Scale 11.

- MMSE: Mini-Mental State Examination.

- RAVLT_immediate: Rey Auditory Verbal Learning Test (sum of scores from 5 first trials).

- Target:

- DX_bl: Subject's diagnosis, i.e., the target variable of which we want to gain a deeper understanding. We built models whose goal is to predict the value of this variable based on the values of other features. The target variable can result in any of the following five values: CN (Cognitive Normal), EMCI (Early Mild Cognitive Impairment), LMCI (Late Mild Cognitive Impairment), SMC (Significant Memory Concern) and AD (Alzheimer's Disease).

Those features are measured by techniques that are able to assess some indicators of whether the individual might be at risk of development, or, has already developed AD symptoms.

The cognitive tests allow the examiner to obtain an overall sense of whether a person is aware of the symptoms, the surrounding environment, whether he/she can remember a short list of words, follow some instructions and do simple calculations. Cognitive tests are able to measure cognitive decline in a direct and quantifiable manner. However, the cognitive decline is one of the latest to become abnormal. This is because the first abnormalities are first noticed at a microscopical scale through the misfolding of a protein called Amyloid beta. These are followed by changes at larger scales, such as loss of the neurons myelin sheath, neuron death, visible atrophy in MRI scans and finally cognitive decline.

Cognitive tests, however, have several limitations that affect their reliability and those are related to remembering them if taken several times, might have floor or ceiling effects, which means that many subjects might score the highest/lowest score possible, and can be biased, as they are undertaken by a human expert who might be influenced by prior knowledge of the subject's cognitive abilities [33].

Magnetic resonance imaging (MRI) is a technique used to quantify by measuring the volume of gray matter - GM (consisted of nerve cells) and white matter WM (fibres connecting the nerve cells). Atrophy is indicated by the loss of volume between one scan and other follow-up scan. It is caused by the death of neurons in regions affected. Quantification of atrophy with MRI is a very important parameter as it is widely available and non-invasive good indicator of progression of MCI to dementia $[34,35,36]$. 
The Positron Emission Tomography (PET) enables researchers to track the concentration of abnormal proteins (amyloid and tau) since the contrast agent (containing the tracer) spreads throughout the brain and binds to abnormal proteins. PET scans can be of several types, depending on the cellular and molecular processes that are being measured. Fluorodeoxyglucose (FDG) PET can be used to measure cell metabolism. Neurons that are about to die show reduced metabolism, so FDG PET is an indicator of neurodegeneration. AV45 PET is used to measure the levels of abnormal proteins such as amyloid-beta. The errors in Amyloid-beta 3D structure (misfolding) is thought to be one of the causes of $\mathrm{AD}$ since its high levels lead to neurodegeneration and cognitive decline. The basic limitation of PET scans is that the patient is exposed to ionizing radiation, which limits the number of scans they can take in a specific time interval [34,37].

The APOE gene provides instructions for producing a protein called apolipoprotein $\mathrm{E}$, and that is why the gene expression measurement is an important technique by which the activity of APOE gene can be quantified. This protein aids the formation of lipoproteins by combining with fats (lipids) in the body. There are at least three slightly different versions (alleles) of the APOE gene. The major alleles are e2, e3, and e4. The $\mathbf{e} 4$ version of the APOE gene is believed to increase an individual's risk for developing AD. People who inherit one copy of the APOE e4 allele have an increased chance of developing the disease, those with two copies of the allele are at even greater risk. In TADPOLE data set, an information about individual's number of present e4 alleles $(0,1$ or 2$)$ is available. However, not necessarily the individuals with $\mathrm{AD}$ have the APOE e4 allele, and also not all individuals who have this allele will develop AD [38].

\subsection{Data set preprocessing}

\subsubsection{Incomplete data}

Figure 3 shows that all subjects contain personal information and diagnosis, but not all of them have data for all the other parameters. All subjects with incomplete data have been detected since incomplete data can cause a lot of troubles in the process of data analysis and building the intelligent models later described in this section. Since the removal of all subjects where data for at least one attribute is missing causes significant information loss, an attempt is be made to use the leverage of some imputation techniques.

For example, only 2,118 subjects have data for the AV45 attribute. Removing all subjects (rows) that contain any missing data from our data set results into new data set with 1,121 entries.

Therefore, it is decided to remove all subjects that miss data for more than 6 features, and then perform data augmentation by using the imputation techniques later described in this section. The initial data removal resulted in 9,592 subjects left for further analysis.

\subsubsection{Redundant features}

The redundancy analysis of the features is crucial step for appropriate experimental setup. Taking a closer look at the PTRACCAT column in Figure 4, it can be perceived that almost $93 \%$ entries have value 'white'. It means that this attribute 
does not provide enough information about possible racial predisposition for the disease. Most of the subjects belong to same racial group and only few belong to other groups, so this feature is be excluded from further analysis. Also PTID attribute is omitted because it is an identification number for each subject and has no meaning for the models we intend to build.

\subsubsection{Categorical features encoding}

The attributes PTGENDER (Male/Female) and DX_bl (CN, EMCI, LMCI, $\mathrm{SMC}$, and $\mathrm{AD}$ ) are of categorical data type. Simply encoding the attribute 'Male' with the value 1 while 'Female' with 0 , would lead to increase the weight of Male compared to that of Female. This does not make sense since both variables need to be treated equally by the model to predict accurate results. To achieve equality one-hot encoding is used for encoding the PTGENDER attribute. This encoding is appropriate for categorical data where no relationship exists between categories. It involves representing each categorical variable with a binary vector that has one element for each unique label and marking the class label with a 1 and all other elements 0 .

Considering the target attribute DX_bl, it can be seen that there is a certain order related to its values. The values can be ranked from $\mathrm{CN}$ to $\mathrm{AD}$, based on the subject's neuropsychological disorder. Therefore, Label encoding has been used to simply convert labels to integer values in ascending order.

\subsubsection{Correlation analysis}

Assuming correlation between two features, it means that one of them does not contribute to better representation of the information for the model to be learned, thus it can be omitted. Heat map representation as shown on Figure 5 has been used to represent the linear correlations between the features in the data set used for this research.

The map provides a strong negative correlation of -1 between PTGENDER_Male and PTGENDER_Female. This coefficient does not provide anything relevant since both features are discrete and represent same category of data, so it will be ignored.

On the other hand, a trend of pretty high coefficients can be noticed between ADAS11 and other cognitive tests results. In fact, highest negative coefficient is between ADAS11 and MMSE (-0.76) and highest positive coefficient is between ADAS11 and CDRSB (0.73). It is possible that this feature does not provide any new information. It seems like it contains repetitive information from other tests. To determine the correlation, the coefficient alone is not sufficient. Additionally, graph-based representation (Figure 6) is used between the two features to better understand the dependency.

From the plot a kind of linear dependency between these two features can be perceived. Plots of ADAS11 and CDRSB/RAVLT immediate show something similar too. Considering the analysis, we assume that ADAS11 does not provide any new information, thus this feature is redundant and can be omitted from the final data set. 


\subsubsection{Missing values imputation}

Discarding entire rows that contain missing values comes at the price of losing data which may be valuable, even though it is incomplete. In order to obtain the maximum information that the data set is providing, a missing values imputation is performed. Each imputation algorithm uses some kind of estimation to obtain missing values, but that does not mean that the value is $100 \%$ estimated correctly. This can cause noise and bias problems in the data set and that is why it should be used with caution.

One type of imputation algorithm is univariate, which imputes values in the $i-t h$ feature dimension using only non-missing values in that feature dimension. Missing values can be imputed with a provided constant value, or using the statistics (mean, median or most frequent) of the particular column. This technique is also referred as simple imputing. By contrast, multivariate imputation algorithms use the entire set of available feature dimensions to estimate the missing values. These algorithms model each feature with missing values as a function of other features, and use that estimate for imputation. This technique is also referred as iterative imputing.

The goal is to compare different estimators to see which one is most effective for the data set. First, a subset of all rows with non missing value was extracted and its score was estimated. After that, a single value was randomly removed from each row and after iterative imputation of the missing values using different estimators, a score was estimated for each one of them.

\subsubsection{Imbalanced targets distribution}

Figure 8 presents the targets distribution, i.e. the distribution of the five different classes considering the data set.

It can be noticed that there is a huge disproportion between the class with most values (LMCI) and class with least values (SMC). The aim is to create approximate uniform distribution of targets, such that each class will have similar number of instances. An undersampling of LMCI targets will be performed, combined with oversampling of other four classes.

Because the sampling processes mix original samples and artificially created ones, in order to be sure that the data set is not biased, first the data set is split into training (70\%) and testing (30\%) subsets and then separate sampling is performed into each one of them. Couple of different oversampling algorithms were tested combined with Random undersampling. The only exception is SMOTETomek algorithm which already combines methods for both oversampling and undersampling.

\subsection{Machine Learning}

Choosing the most optimal algorithm for solving the problem at hand depends on many factors like: size of the training data, training time, linearity, and number of features. The data set used is medium sized with average number of features allowing to experiment with more complex algorithms. Hence, two types of classifiers are built, one by using Random Forest and the other by using XGBoost algorithm. XGBoost has several advantages, among which it requires less feature engineering, meaning there is no need for scaling and normalizing data. It is less prone to overfitting if the hyperparameters are tuned properly. To validate the trained classifiers properly, 5 -fold cross-validation is performed on the training set. 
One of the crucial steps in building ML model is tuning its hyperparameters the arguments that can be set before training and which define how the training is done. These parameters are tunable and can directly affect how well a model trains. Thus, in order to achieve maximal performance, it is important to understand how to optimize them. To find the best combination of values for hyperparameters for both the Random Forest and XGBoost model, a range of values for every parameter has been defined and then Grid Search has been used which evaluates all combinations and chooses the best one.

\subsection{Models evaluation}

Different performance metrics are used to evaluate the intelligent models built as described in the previous section.

Accuracy is considered a good measure for this research since it is reliable metric when the target variable classes in the data are nearly balanced. Precision is also considered informative measure since it tells the proportion of subjects diagnosed with one of the diagnosis (predicted class), that actually had that diagnosis (real class). Recall is used to measure the proportion of subjects that actually had particular diagnosis (real class) was diagnosed by the model to have that diagnosis (predicted class). Specificity is also used to measure the model's ability to correctly generate a negative result for subjects who do not have the condition that is being tested. A high-specificity model will correctly rule out almost everyone who does not have the disease and will not generate many false-positive results. A model with high sensitivity but low specificity, results in many individuals who are disease free being told of the possibility that they have the disease, and are then subject to further investigation. The last metric used is F1-score, presenting the harmonic mean of both the precision and recall, and thus is considered to be very powerful for the problem at hand.

\subsection{Explainable Machine Learning}

In the context of ML systems, interpretability is the ability to explain the model's output. When a model is built, one need to be able to understand how it is making the predictions. Feature importance helps in estimating how much each feature of the data contributed to the model's prediction. In this research the feature importance is considered in terms of Shapley values by using the SHAP (SHapely Addictive exPlanations) method [39].

SHAP provides two aspects of model's interpretability:

1 The first one is global interpretability - the collective SHAP values can show how much each predictor contributes, either positively or negatively, to the target variable.

2 The second aspect is local interpretability - each observation gets its own set of SHAP values. This greatly increases its transparency.

\section{Results}

\subsection{Data preprocessing}

Considering the data imputation methods applied, as it can be seen from the bar chart presented in Figure 7, Extra Trees Regressor and Bayesian Ridge estimated 
values are closest to the original data. Both of them are multivariate algorithms. On the other hand, univariate algorithms using mean and median failed to do the estimation very accurately, which is somewhat expected considering the fact that the data set contains features represented by sensitive data values where simple average does not solve the problem.

In addition, k-Nearest Neighbors imputation approach was performed. By default, a euclidean distance metric that supports missing values is used to find the nearest neighbors. Each missing feature is imputed using values from k-nearest neighbors that have a value for the feature. Although this type of imputation showed better results than the simple imputation algorithms, it did not surpass the iterative imputation using Extra Trees Regressor.

Considering the results from the comparison, the Extra Trees Regressor was chosen to impute the missing values in the original data set. After that, several such regressors with different number of estimators were tested on the original data set. Experiments have shown that Extra Trees Regressor with 100 estimators most effectively approximates the missing values.

Evaluation results suggest that the SMOTE oversampling algorithm provides the best results as shown in Figure 9, although the difference from one to another is very minor. Another area to explore would be to test different values of the k-nearest neighbors selected in the SMOTE procedure when each new synthetic sample is created. The default is $\mathrm{k}=5$, although larger or smaller values will influence the types of samples created, and in turn, may impact the performance of the model. The repeated and stratified $\mathrm{k}$-fold evaluation showed that the oversampling process is most successful when the number of k-nearest neighbors is equal to 6 as depicted in Figure 10.

The pie charts in Figure 11 represent the number of targets divided by classes in both training and testing subsets after the sampling techniques were applied. It can be seen that they are now almost uniformly distributed.

Completing this step, the data preprocessing phase is finished and the data set is ready to serve as a source for building the intelligent models. The resulting data set after the preprocessing consists of 13,235 subjects.

\subsection{Random Forest model evaluation}

The confusion matrix for the Random Forest model is presented on Figure 12. One of the model's anomalies is the slightly increased number of AD patients predicted with a LMCI diagnosis. The same problem occurs with LMCI patients that are predicted with an AD diagnosis. This tells that perhaps there are some features that bring confusion between these two diagnoses. In addition, there is an increased trend into false negatives of the SMC class, but considering the fact that serious amount of the SMC subjects were created artificially, this phenomenon is somewhat understandable.

Table 1 shows detailed report of the evaluation metrics for the Random Forest model. Through LMCI's precision and recall, it can be noticed that the false predictive relationship between LMCI and AD subjects quite negatively affected the model's metrics. Lower recall value at SMC is due to false categorization of SMC patients as EMCI and CN. Besides this two problems, it can be stated that the model metrics are pretty stable, without abrupt deviations. 


\subsection{XGBoost model evaluation}

XGBoost classifier performed slightly better than the Random Forest classifier as shown in Figure 13. Table 1 shows that XGBoost managed to guess more subjects with their correct diagnosis, thus it represents more accurate model than the Random Forest model. Majority of subjects lay on the main diagonal. Trends about groups of false predictions analysed before are present here as well, however, with smaller intensity. Number of subjects predicted as AD while having LMCI diagnosis and vice versa stand out again, together with the distribution between $\mathrm{CN}$ and EMCI of false predicted SMC subjects.

XGBoost model managed to achieve balanced accuracy of 0.84 . Other metrics are also very satisfactory, deviating from 0.8 to 1.0 .

\subsection{Random Forest model interpretability}

\subsubsection{Global interpretability}

The following Figure 14 presents how high is each feature influence (variables importance) on each group of targets in average.

It can be seen that CDRSB feature has highest importance in defining outcome for one subject, and this is valid for each class of subjects. All features that describe cognitive tests results are generally ranked on the top. Results from MMSE have significant high impact on the AD diagnosis, comparing to other classes.

Note that the gender features are at the very bottom, which means their values influence the outcome least. This information tells us that probably there is not a gender predisposition for obtaining Alzheimer's disease. Another interesting thing to observe is the ranking of APOE4 feature. Many studies [40, 41] say that e4 alleles of the APOE gene can be a predisposition for dementia, but not necessarily. From this result, it can be confirmed that the APOE gene does not act as a decisive factor for having a diagnosis.

We can also take a closer look of whether one feature has positive or negative impact on the output and how high is it really, by looking at the summary plot for each diagnosis. The plot is made of all the dots in the train data.

Figures 15 and 16 depict the summary plots of variables importance for $\mathrm{CN}$ and LMCI class diagnosis.

On the summary plot of CN class, we can notice that low values of CDRSB have very high positive impact determining this diagnosis. Additionally, high values of MMSE and RAVLT_immediate also tend to have high positive impact. Contrary to already mentioned conclusions, it can be noticed that younger subjects tend to decline from this diagnosis. Although the natural way of thinking is that neurological diseases affect only older people, that is not the truth. In addition, from TADPOLE data set it can be concluded that women are more inclined to neurodegeneration.

If we compare this analysis with the LMCI summary plot, it can be noticed slightly different color allocation. For example, very high and very low CDRSB values impact the model's output negatively. Here, high values of RAVLT_immediate have negative impact, which is somewhat expected. Higher values for MRI measurements tend to influence on this class as an output negatively too.

The summary plot for AD class shown in Figure 17 shows significantly opposite trends than the CN class. High CDRSB values impact positively, while high MMSE 
results tend to impact on the output for this class negatively. Also, low values for FDG have a positive impact for this class, contrary to the CN class where low values had slightly negative impact. Important thing to notice is how high education influences negatively on the output to a large extent.

Even more information can be uncovered examining the partial dependence plot of one feature. This plot shows the marginal effect two features have on the predicted outcome. Once the first feature is chosen, the second is automatically selected depending with which one the first interacts most. These plots are also class specific. Most of the plots show more complex correlation, or no correlation at all, but those that are most relevant will be examined.

A clear high correlation between the features WholeBrain and MidTemp can be seen from Figure 18. Values of MidTemp increase together with the WholeBrain's. Although, there is clear correlation between them, it can not be concluded how they influence the predicted outcome. It seems like data points from above $\mathrm{y}=0$ are reflected below, which means that we have cases where approximately same set of values influences one outcome positively and another negatively.

Figure 19 presents a nonlinear positive correlation between CDRSB and the AD diagnosis. There is also an obvious relationship between CDRSB and MMSE implicating that subjects with higher MMSE tend to have lower CDRSB and these values affect the model's output slightly negative. Mid to lower values of MMSE give positive impact on this diagnosis.

Last but not least, Figure 20 presents an example of complex but slightly negative correlation between FDG and predicted output, the AD class. As the FDG values increase, the positive impact on the output decreases and turns into negative. There is also relationship between FDG and CDRSB exposing that subjects with higher CDRSB tend to have lower FDG and vice versa.

\subsubsection{Local interpretability}

By isolating a single subject, it can be explained graphically how features influence the subject to become labeled with particular class. One subject belongs to all classes with different probabilities and is labeled with the class with highest probability. Let's consider a subject that was correctly predicted with CN diagnosis as shown in Figure 21. The graph presents an output value or $\mathbf{f}(\mathbf{x})$ which is prediction probability for the particular observation, and base value or $\mathbf{E}[\mathbf{f}(\mathbf{x})]$ which is the value that would be predicted if any features are not known for the current output (mean prediction).

It can be seen that the prediction starts from the base value for $\mathrm{CN}$ class and most of the features push the value to the right, increasing the probability for this subject to belong to this class. On the other side, for Figure 22 it can be seen that the plot for this subject to belong to SMC class is not straightforward at all. Most of the features have high negative impact and that is the reason why the subject's probability to be labeled as SMC is so small.

This interpretation can be used to analyse the trends of false predicted subjects mentioned before. The features influence is examined on the predicted and true class of randomly selected subject belonging to the particular trend. On each of the figures 23, 24, 25, and 26 the top plot represents features influence of subject's predicted class and the bottom one of subject's true class. 
First to be discussed is a subject with LMCI diagnosis, predicted as AD. From Figure 23 it can be noticed that results from cognitive tests greatly contribute into the false prediction. On the top plot, CDRSB, MMSE and RAVLT_immediate significantly push the probability value for the AD class to the right, while only few features contribute trivially to lower this value. It can be concluded that main features responsible for the false predictions are results from cognitive tests. The opposite thing happens on the bottom plot of LMCI class. Features mentioned before, push the probability to the left, minimizing its value to 0.17 .

Next, a subject with SMC diagnosis, predicted as EMCI is chosen and shown on Figure 24. Although the probability of false predicted class here is smaller then the previous case and more features contribute to lower it, still the probability of the correct class is significantly small. Low value for CDRSB, combined with high value for MidTemp, affects the output crucially in favor of EMCI. Note that FDG has positive impact in both cases, but it is slightly greater in the predicted class.

On figures 25 and 26 it can be noticed that difference between class probabilities is remarkably minor. These figures represent subject with SMC diagnosis, predicted as CN and subject with AD diagnosis, predicted as LMCI, respectively. In the first case, all features that influence the predicted output positively, have negative impact on the true class and vice versa. Only CDRSB has positive impact in both classes, although it is larger in the predicted one. In the last case, RAVLT_immediate and Entorhinal are main features that lead to false prediction, having positive impact on the predicted class and negative on the one that is true. Also, FDG's positive impact is crucially higher in favor of LMCI class.

\subsection{XGBoost model interpretability}

\subsubsection{Global interpretability}

The ranking of features presented in Figure 27 is pretty similar to the previous Random Forest model. Analysing the plot, it can be noticed that CDRSB leads by far the most in the impact on this model's output. Interesting thing to notice is the ascent of AGE and PTEDUCAT, while in the previous model AGE was ranked somewhere in the middle and PTDEDUCAT was between lowest ranked features. Another thing worth mentioning is the position of FDG and APOE4. Both of them were ranked higher in the previous model, but here we can see that APOE4 has smaller impact than the gender feature. Since PTGENDER_Male and PTGENDER_Female are highly correlated and basically represent one feature, impact values of PTGENDER_Female are omitted here.

Looking at the color distributions at some features, it can be seen that MMSE value impacts most on the $\mathrm{CN}$ subjects. On the other hand, subject's age has most influence for LMCI class. For the AD class, the impact of the gender feature is insignificant. If we take a closer look on the green parts of each feature, we can notice that cognitive tests have highest impact on the CN class, while other features impact the output for this class in smaller amount.

Analysing features importance for CN class separately presented in Figure 28, we can confirm some of the statements we made earlier. For example, once again we confirm that low values of CDRSB have significant positive impact for predicting this class. While other MRI measures do not give useful information on how they 
affect the model's output, the WholeBrain feature have serious negative impact for higher values and vice versa. The same statement applies to Hippocampus too, but not to that extent. Highly negative impact for lower values of AGE feature can be observed. This trend was present on the same plot for the previous model too, but here it is more clearly shown.

For the LMCI class, shown in Figure 29, the situation is slightly different, as expected. Low AGE values are distributed on the positive axis now, denoting positive impact for this class.

Contrary to the previous situation, here it can be seen that lower values for CDRSB have highly negative impact on this class, high values do not have any impact at all, while mid values have positive impact. It is very likely that high values are going to be distributed on the positive axis for the $\mathrm{AD}$ class. Other features (Hippocampus, RAVLT_immediate, FDG and MidTemp) have similar types of distributions denoting negative impact on model's output for higher feature's values.

Figure 30 shows that high CDRSB values indeed represent high positive impact for AD class. In opposite, higher MMSE values have significant negative impact. Looking at the PTEDUCAT feature, it can be seen that higher education has valuable negative impact on the Alzheimer's diagnosis. Also, the APOE4 distribution indicates that subjects with zero e4 alleles have less chance of being labeled with $\mathrm{AD}$ than those with one or two. It can also be confirmed that subjects with lower values of FDG tend to be diagnosed with this diagnosis.

Last thing that needs to be covered from the global interpretability of the XGBoost model are the dependence plots. Comparing to the previous Random Forest model's plots, there are some more interesting trends and dependencies to be shown here.

First, the marginal effect FDG and CDRSB have on the predicted outcome is analysed for three different classes (EMCI, LMCI, AD) on Figure 31. On all three plots we can notice the correlation between FDG and CDRSB, as subjects with lower FDG tend to have higher CDRSB results. The plot color changes progressively from yellow to blue, as we move on the $\mathrm{x}$-axis from 0.6 to 1.8. But what fascinates the most is how the positive correlation that they have with the outcome gradually evolves into negative one, as the disease stages move from cognitive normal to Alzheimer's disease.

In Figure 32, it can be seen that younger subjects with two alleles e4 of APOE gene have bigger chances of having an Alzheimer's disease. This represents intriguing information about the genetic predisposition of the disease, since we can see that APOE4 and AGE are correlated somehow. In addition, high values for AGE and zero e 4 alleles have negative impact on this class. Contrary, one e4 allele and higher age values have a positive impact.

Last but not least, there is pretty complex correlation between RAVLT_immediate and MidTemp and they also influence the output in non-straightforward way, thus a couple of statements can be made from Figure 33. Diving deeper into the plot, it can be seen that subjects with lower values for RAVLT_immediate tend to have lower values for MidTemp too and they have mild positive influence for LMCI class. On the other hand, higher values for the cognitive test correlated with mid to high MidTemp values tend to have negative impact. 


\subsubsection{Local interpretability}

Opposite to the previous analysis of local interpretability for the Random Forest model, now a randomly chosen subject whose diagnosis was predicted correctly as $\mathrm{AD}$ is considered. Observing the waterfall plot in Figure 34 for the correctly predicted class and the LMCI class in Figure 35, the following conclusions can be derived.

As it is expected for the AD class, almost all features have positive impact, except the MidTemp with significant weak negative impact. The prediction is straightforward with pretty high probability. On the other side, the prediction for the LMCI class for the same subject have pretty mixed up feature impacts. We can see that some of the features influence positively in the favor of LMCI class, but other features have higher negative impacts together, thus the subject's probability to belong to this class is pretty low. In both cases, the CDRSB impact is crucial.

Next, the false predictive trends are analyzed once again, but now with different randomly chosen subjects belonging to the respective classes. For each of the figures $36,37,38$, and 39 top plot represents the predicted class, while the bottom plot is for the true class.

On Figure 36, a subject with LMCI diagnosis, predicted as AD is shown. It can be noticed that the difference between probability for the predicted class and the true one is slightly big. Besides CDRSB, in this case MMSE and AGE play significant role in increasing one's probability and decreasing the other. It seems like most of the patient's medical measurements indicate an AD instead of LMCI.

Figure 37 shows individual plots of a subject with SMC diagnosis, predicted as EMCI. Even bigger probability difference occurs here. On the first plot, almost all features influence positively in favor of EMCI diagnosis. The problem with many false predictions correlated to the SMC class may be because of the initially deficient subset of targets we were dealing with at the beginning. The low CDRSB value influences the most against the true class and in favor of the predicted one, which was indeed proven (higher CDRSB values tend to correlate with more devastating diagnoses).

In the next two figures 38 and 39, plots of a subject with SMC diagnosis, predicted as CN and subject with AD diagnosis, predicted as LMCI are shown. The probability difference in both cases is slightly smaller than before, but it is still pretty high. In the first case, CDRSB has positive impact for both classes, but for the predicted one it is slightly higher. MidTemp and AGE are playing the main role here in deciding the final output. In the last Figure 39, MRI measurements have greatest impact on the subject. It can be seen that they have serious positive impact for the predicted class, in the same time decreasing the probability for the true class.

\section{Discussion}

The results are summarized in Table 2. For summarizing the categorical metrics, weighted average representation has been used. In addition, the times required to train the models are compared, as well as times required to predict the targets of the testing data set. Although the difference is not huge, it still can be confirmed that the XGBoost algorithm performs better classification of subjects and better 
distinguishes between different types of neurodegenerative diagnoses, indicating that it is more reliable and accurate than his opponent in this research - the Random Forest model.

The global interpretability of both intelligent models provided exceptional conclusions that were deeply explained in the previous section. However, not all of the conclusions were comprehensively discussed and thus, the features influence on predicting a particular class has been summarized in Table 5 and 6 for both of the Random Forest and XGBoost models correspondingly. Both tables provide detailed scheme of the positive (+), negative (-), and undefined (X) influence the particular value of the feature has on predicting the particular class. This scheme can be used as additional knowledge for the physicians and other related experts when they are making conclusions over the diagnosis for particular patient.

Considering both of the tables 5 and 6 , it can be once again confirmed that XGBoost provides higher explainability of the problem, since there are less undefined influence status in the XGBoost global interpretability table. This is especially true for the MRI features. Luckily, there is a low level of inconsistency between the unveiled influence provided in both of the tables. It is very rare that one model is contradictory to the other, e.g., the high education positive or negative influence on the SMC class is suspicious, and also APOE4 2 influence on the EMCI class is a matter of question whether it is positive or negative. To achieve better understanding, both tables were merged into a single table unveiling the contribution to the undefined influence in each of them.

The newly merged Table 7 presents the best contributions of each model, masking the two contradictory influence as undefined.

Table 2 shows the times required for the models to be trained and to predict the entire test set. The XGBoost classifier requires more time to be trained since it uses gradient boosting algorithms in background, but its predictions are slightly faster than the ones of Random Forest classifier. Benefits obtained in terms of model's exactness and validity for the time difference are more than worthy.

It can be concluded that XGBoost is more optimal algorithm for dealing with Alzheimer's prediction problem using the particular data set. Table 3 presents a comparison of our XGBoost model with other models trained on the same original TADPOLE data set [42]. As shown it can be perceived that our model is ranked second, and if a performance time is included as trade-off, then our model would be on the top of the list.

Considering the popular $\mathrm{AD}$ research, mostly considered those published in the latest years, Table 4 presents an interesting review not only of the obtained results and the data set used, but also on the experimental setup performed in each of the reported experiments. High accuracy metrics are reported, however most of them show no clear evidence on appropriate methods for data preprocessing, missing data imputation, hyperparameters tuning, appropriate split of train and testing set, and the data sets used are limited to at most nearly two thousand patients. The most remarkable research among the presented is the one published in [43] in which very comprehensive mathematically supported approach is presented with special attention on avoiding over/underfitting problems. 


\section{Conclusion}

The main objective of the study presented is to put at test the existing hypothesis regarding the causes and indicators of Alzheimer's disease. At the beginning of the research, four hypothesis were established considering the existing published literature. Hereafter, a large data set was obtained containing various types of features considering the lifestyle, personal information, medical analysis and cognitive tests of 12741 individuals (subjects). The data set was used in accordance with ethics and after obtaining a special permission for research goals.

To test the established hypothesis, intelligent models were built by following a ML approach. The high performance of the models (Random Forest and XGBoost) was used in advantage of explainable ML methods able to interpret the relations among the various features and therefore, to derive conclusion over the causes and indicators of the Alzheimer's disease.

The most important contribution from this research is the established scheme presented in Table 7. This table provides a summarized features positive or negative influence on diagnosing each class, according to the global interpretability of both of the intelligent models built.

Thus, the comprehensive analysis of the features importance considering both the global and local interpretability, led to the following important conclusions regarding the previously established hypothesis:

- Cognitive tests, especially CDRSB, have greatest influence on one model's outcome regarding all of the classes.

- Higher CDRSB values tend to correlate with more devastating diagnoses.

- Subject's gender impact slightly on the model's outcome.

- There is a unique combination of MRI indicators influence for each of the diagnosis, and this might be really interesting for the physicians.

- APOE gene is not always a decisive factor in determining a diagnosis.

- Important thing noticed is high and low education influence positively on determining the extremely distance diagnoses, $\mathrm{CN}$ and $\mathrm{AD}$, correspondingly.

- It also can be noticed that results from cognitive tests greatly contribute into the false prediction.

- It can also be confirmed that subjects with lower values of FDG tend to be diagnosed with AD, but also with LMCI.

- Subjects with lower FDG tend to have higher CDRSB results.

- Fascinating is how the positive correlation that FDG and CDRSB have with the outcome gradually evolves into negative one, as the disease stages move from $\mathrm{CN}$ to $\mathrm{AD}$.

- Younger subjects with two alleles e4 of APOE gene also have bigger chances of developing $\mathrm{AD}$.

- High values for AGE and zero e4 alleles have negative impact on developing $\mathrm{AD}$.

- One e4 allele and higher AGE values have a positive impact on developing $\mathrm{AD}$.

Considering the conclusions, all the hypothesis are rejected, showing that $\mathrm{AD}$ is complex disease that cannot be initiated by genetics alone, nor the gender, nor the age, and nor the lack of education. 
This research is believed to have big influence on the future directions for understanding $\mathrm{AD}$ as well as large influence on the future researchers regarding the usage of explainable ML methods to unveil new knowledge also in other diseases data.

Such analysis are expected to affect also the medical approaches for on-time diagnosing and therefore, proper treating with the aim to slow down the progression of the disease, and thus reduce the damage that this disease causes to a person, as well as to his surrounding.

\begin{abstract}
Acknowledgements
The motive for starting a research about Alzheimer's disease, working on it fully committed until its wording into this paper, lies in the cruel fate of my father, who was diagnosed with this disease in the golden period of his life. Thus, I dedicate this paper in his honour. My heart will be full if this research helps at least in minor way in developing approach for controlling this disease. I would like to thank my mother, brother and friends for standing by me and giving me support, not only for completing this paper, but in general through life. Last but not least, I would like to thank co-authors Monika Simjanoska and Tome Eftimov for the incredible collaboration and the opportunity provided to learn from remarkable minds like them. - B.B.
\end{abstract}

\title{
Funding
}

This publication is funded by the Slovenian Research Agency (research core funding No. P2-0098 and project No. Z2-1867), and the European Union's Horizon 2020 research and innovation programme under grant agreement 863059 (FNS-Cloud, Food Nutrition Security) and under grant agreement 101005259 (COMFOCUS). Data collection and sharing for this project was funded by the Alzheimer's Disease Neuroimaging Initiative (ADNI) (National Institutes of Health Grant U01 AG024904) and DOD ADNI (Department of Defense award number W81XWH-12-2-0012). ADNI is funded by the National Institute on Aging, the National Institute of Biomedical Imaging and Bioengineering, and through generous contributions from the following: AbbVie, Alzheimer's Association; Alzheimer's Drug Discovery Foundation; Araclon Biotech; BioClinica, Inc.; Biogen; Bristol-Myers Squibb Company; CereSpir, Inc.; Cogstate; Eisai Inc.; Elan Pharmaceuticals, Inc.; Eli Lilly and Company; Eurolmmun; F. Hoffmann-La Roche Ltd and its affiliated company Genentech, Inc.; Fujirebio; GE Healthcare; IXICO Ltd.; Janssen Alzheimer Immunotherapy Research Development, LLC.; Johnson Johnson Pharmaceutical Research Development LLC.; Lumosity; Lundbeck; Merck Co., Inc.; Meso Scale Diagnostics, LLC.; NeuroRx Research; Neurotrack Technologies; Novartis Pharmaceuticals Corporation; Pfizer Inc.; Piramal Imaging; Servier; Takeda Pharmaceutical Company; and Transition Therapeutics. The Canadian Institutes of Health Research is providing funds to support ADNI clinical sites in Canada. Private sector contributions are facilitated by the Foundation for the National Institutes of Health (www.fnih.org). The grantee organization is the Northern California Institute for Research and Education, and the study is coordinated by the Alzheimer's Therapeutic Research Institute at the University of Southern California. ADNI data are disseminated by the Laboratory for Neuro Imaging at the University of Southern California.

Availability of data and materials

Data used in the preparation of this article were obtained from the Alzheimer's Disease Neuroimaging Initiative (ADNI) database (adni.loni.usc.edu). The ADNI was launched in 2003 as a public-private partnership, led by Principal Investigator Michael W. Weiner, MD. The primary goal of ADNI has been to test whether serial magnetic resonance imaging (MRI), positron emission tomography (PET), other biological markers, and clinical and neuropsychological assessment can be combined to measure the progression of mild cognitive impairment $(\mathrm{MCl})$ and early Alzheimer's disease (AD). For up-to-date information, see www.adni-info.org.

Ethics approval and consent to participate

As per ADNI protocols, all procedures performed in studies involving human participants were in accordance with the ethical standards of the institutional and/or national research committee and with the 1964 Helsinki declaration and its later amendments or comparable ethical standards. More details can be found at adni.loni.usc.edu. This article does not contain any studies with human participants nor animals performed by any of the authors.

Competing interests

The authors declare that they have no competing interests.

Consent for publication

Authors proclaim that all terms of the data use agreement are accepted and included in the manuscript. The manuscript has been sent to ADNI Data and Publication Committee and it has been approved for publishing. Authors acknowledge that all images are entirely unidentifiable and there are no details on individuals reported within the manuscript.

Authors' contributions

B.B. worked on creation of the draft and final manuscript including data preprocessing stages, models' implementation and evaluation followed by initial analysis of their interpretability. T.E. worked on editing the draft manuscript and discussing the obtained results and conclusions. M.S. worked on creation of the manuscript, organization of the results, and on providing in-depth discussion over the achieved conclusions from the research. 


\section{Author details}

${ }^{1}$ Faculty of Computer Science and Engineering, Ss. Cyril and Methodius University, Rugjer Boshkovikj 16, 1000 Skopje, N. Macedonia. ${ }^{2}$ Computer Systems Department, Jozef Stefan Institute, Jamova cesta 39, 1000, Ljubljana, Slovenia. ${ }^{3}$ iReason, LLC, Skopje, N. Macedonia. ${ }^{4}$ Data used in preparation of this article were obtained from the Alzheimer's Disease Neuroimaging Initiative (ADNI) database (adni.loni.usc.edu). As such, the investigators within the ADNI contributed to the design and implementation of ADNI and/or provided data but did not participate in analysis or writing of this report. A complete listing of ADNI investigators can be found at:

http://adni.loni.usc.edu/wp-content/uploads/how_to_apply/ADNI_Acknowledgement_List.pdf.

\section{References}

1. Reitz, C., Brayne, C., Mayeux, R.: Epidemiology of alzheimer disease. Nature Reviews Neurology 7(3), 137-152 (2011)

2. Bird, T.D.: Alzheimer disease overview. GeneReviews $\mathbb{R}[$ Internet] (2018)

3. Ballard, C., Gauthier, S., Corbett, A., Brayne, C., Aarsland, D., Jones, E.: Alzheimer's disease. The Lancet 377(9770), 1019-1031 (2011). doi:10.1016/S0140-6736(10)61349-9

4. Marcus, E.-L., Kaufman, Y., Cohen-Shalev, A.: Creative work of painters with alzheimer's disease. Harefuah 148(8), 548-53 (2009)

5. Association, A., et al.: 2018 alzheimer's disease facts and figures. Alzheimer's \& Dementia 14(3), 367-429 (2018)

6. Scahill, R.I., Schott, J.M., Stevens, J.M., Rossor, M.N., Fox, N.C.: Mapping the evolution of regional atrophy in alzheimer's disease: unbiased analysis of fluid-registered serial mri. Proceedings of the National Academy of Sciences 99(7), 4703-4707 (2002)

7. Yang, E., Farnum, M., Lobanov, V., Schultz, T., Raghavan, N., Samtani, M.N., Novak, G., Narayan, V., DiBernardo, A., Initiative, A.D.N., et al.: Quantifying the pathophysiological timeline of alzheimer's disease. Journal of Alzheimer's Disease 26(4), 745-753 (2011)

8. Doody, R.S., Pavlik, V., Massman, P., Rountree, S., Darby, E., Chan, W.: Predicting progression of alzheimer's disease. Alzheimer's research \& therapy 2(1), 1-9 (2010)

9. Guerrero, R., Schmidt-Richberg, A., Ledig, C., Tong, T., Wolz, R., Rueckert, D., (ADNI, A.D.N.I., et al.: Instantiated mixed effects modeling of alzheimer's disease markers. Neurolmage 142, 113-125 (2016)

10. Klöppel, S., Stonnington, C.M., Chu, C., Draganski, B., Scahill, R.I., Rohrer, J.D., Fox, N.C., Jack Jr, C.R., Ashburner, J., Frackowiak, R.S.: Automatic classification of mr scans in alzheimer's disease. Brain 131(3), 681-689 (2008)

11. Zhang, D., Wang, Y., Zhou, L., Yuan, H., Shen, D., Initiative, A.D.N., et al.: Multimodal classification of alzheimer's disease and mild cognitive impairment. Neuroimage 55(3), 856-867 (2011)

12. Fonteijn, H.M., Modat, M., Clarkson, M.J., Barnes, J., Lehmann, M., Hobbs, N.Z., Scahill, R.I., Tabrizi, S.J., Ourselin, S., Fox, N.C., et al.: An event-based model for disease progression and its application in familial alzheimer's disease and huntington's disease. Neurolmage 60(3), 1880-1889 (2012)

13. Young, A.L., Oxtoby, N.P., Daga, P., Cash, D.M., Fox, N.C., Ourselin, S., Schott, J.M., Alexander, D.C.: A data-driven model of biomarker changes in sporadic alzheimer's disease. Brain 137(9), 2564-2577 (2014)

14. Jedynak, B.M., Lang, A., Liu, B., Katz, E., Zhang, Y., Wyman, B.T., Raunig, D., Jedynak, C.P., Caffo, B., Prince, J.L., et al.: A computational neurodegenerative disease progression score: method and results with the alzheimer's disease neuroimaging initiative cohort. Neuroimage 63(3), 1478-1486 (2012)

15. Donohue, M.C., Jacqmin-Gadda, H., Le Goff, M., Thomas, R.G., Raman, R., Gamst, A.C., Beckett, L.A., Jack Jr, C.R., Weiner, M.W., Dartigues, J.-F., et al.: Estimating long-term multivariate progression from short-term data. Alzheimer's \& Dementia 10, 400-410 (2014)

16. Durrleman, S., Pennec, X., Trouvé, A., Braga, J., Gerig, G., Ayache, N.: Toward a comprehensive framework for the spatiotemporal statistical analysis of longitudinal shape data. International journal of computer vision 103(1), 22-59 (2013)

17. Lorenzi, M., Pennec, X., Frisoni, G.B., Ayache, N., Initiative, A.D.N., et al.: Disentangling normal aging from alzheimer's disease in structural magnetic resonance images. Neurobiology of aging 36, 42-52 (2015)

18. Paul T Francis, M.S. Alan M Palmer, Wilcock, G.K.: The cholinergic hypothesis of alzheimer's disease: a review of progress. Journal of Neurology, Neurosurgery Psychiatry 66(2), 137-147 (1999)

19. Mudher, A., Lovestone, S.: Alzheimer's disease-do tauists and baptists finally shake hands? Trends in neurosciences 25(1), 22-26 (2002)

20. NHS: Alzheimer's disease - Causes - NHS. https://www.nhs.uk/conditions/alzheimers-disease/causes/

21. Jorm AF, J.D.: The incidence of dementia: a meta-analysis. Neurology 51(3), 728-33 (1998)

22. Stephen Todd, M.R. Stephen Barr, Passmore, A.P.: Survival in dementia and predictors of mortality: a review. International journal of geriatric psychiatry 28(11), 1109-1124 (2013)

23. Liu, L.C.C.K.T.X.H..B.G. C. C.: Apolipoprotein e and alzheimer disease: risk, mechanisms and therapy. Nature reviews 9(2), 106-118 (2013)

24. Bachman DL, L.R.e.a. Wolf PA: Prevalence of dementia and probable senile dementia of the alzheimer type in the framingham study. Neurology 42, 115-19 (1992)

25. Martins, H.E.F.J.e.a. I.: Apolipoprotein e, cholesterol metabolism, diabetes, and the convergence of risk factors for alzheimer's disease and cardiovascular disease. Mol Psychiatry 11, 721-736 (2006)

26. Speck, K.W.B.D.B.J.M.W.T.L.L.E. C.: History of depression as a risk factor for alzheimer's disease. Epidemiology 6(4), 366-369 (1995)

27. J Eric Ahlskog, N.R.G.-R. Yonas E Geda, Petersen, R.C.: Physical exercise as a preventive or disease-modifying treatment of dementia and brain aging. Mayo Clinic Proceedings 86, 876-884 (2011)

28. Janine K Cataldo, J.J.P., Glantz, S.A.: Cigarette smoking is a risk factor for alzheimer's disease: an analysis controlling for tobacco industry affiliation. Journal of Alzheimer's disease 19(2), 465-480 (2010)

29. Samadi, M.S.M.M.e.a. M.: Dietary pattern in relation to the risk of alzheimer's disease: a systematic review. Neurol Sci 40, 2031-2043 (2019) 
30. L., T.S.: Alcohol use and the risk of developing alzheimer's disease. Alcohol research health : the journal of the National Institute on Alcohol Abuse and Alcoholism 25(4), 299-306 (2001)

31. Lye, S.E.A. T.C.: Traumatic brain injury as a risk factor for alzheimer's disease: A review. Neuropsychol Rev 10, 115-129 (2000)

32. Marinescu, R.V., Oxtoby, N.P., Young, A.L., Bron, E.E., Toga, A.W., Weiner, M.W., Barkhof, F., Fox, N.C., Klein, S., Alexander, D.C., et al.: Tadpole challenge: Prediction of longitudinal evolution in alzheimer's disease. arXiv preprint arXiv:1805.03909 (2018)

33. Guy McKhann, M.F.R.K.D.P. David Drachman, Stadlan, E.M.: Clinical diagnosis of alzheimer's disease report of the nincds-adrda work group under the auspices of department of health and human services task force on alzheimer's disease. Neurology 34(7), 939-939 (1984)

34. Clifford R Jack, W.J.J.L.M.S.P.S.A.M.W.W.R.C.P. David S Knopman, Trojanowski, J.Q.: Hypothetical model of dynamic biomarkers of the alzheimer's pathological cascade. The Lancet Neurology 9(1), 119-128 (2010)

35. Martin Reuter, H.D.R. Nicholas J Schmansky, Fischl, B.: Within subject template estimation for unbiased longitudinal image analysis. Neuroimage 61(4), 1402-1418 (2012)

36. Clifford R Jack Jr, W.J.J.R.C.P.M.W.W.P.S.A.L.M.S.P.V.H.J.W.S.D.W.e.a. David S Knopman: Update on hypothetical model of alzheimer's disease biomarkers. Lancet neurology 12(2), 207 (2013)

37. Marta Marquié, C.R.V.I.M.C.E.A.B.L.G.R.W.E.K.C.A.M.M.D.I.M.L.D.e.a. Marc D Normandin: Validating novel tau positron emission tomography tracer [f-18]-av-1451 (t807) on postmortem brain tissue. Annals of neurology 78(5), 787-800 (2015)

38. Kelly, E.B.: Alzheimer's Disease (Genes Disease) (Illustrated Ed.). Chelsea House Publications, New York NY 10001, United States of America (2008)

39. Lundberg, S.M., Lee, S.-I.: A unified approach to interpreting model predictions. In: Guyon, I., Luxburg, U.V., Bengio, S., Wallach, H., Fergus, R., Vishwanathan, S., Garnett, R. (eds.) Advances in Neural Information Processing Systems 30, pp. 4765-4774. Curran Associates, Inc., ??? (2017). http://papers.nips.cc/paper/7062-a-unified-approach-to-interpreting-model-predictions.pdf

40. Meyer, T.J.N.M.e.a. M.: Apoe genotype predicts when - not whether - one is predisposed to develop alzheimer disease. Nature Genetics 19, 321-322 (1998)

41. Kambooh, M.: Apolipoprotein e polymorphism and susceptibility to alzheimer's disease. Human Biology 67(2), 195-215 (1995)

42. EuroPOND and ADNI: TADPOLE Challenge. https://tadpole.grand-challenge.org/

43. Spasov, S., Passamonti, L., Duggento, A., Liò, P., Toschi, N., Initiative, A.D.N., et al.: A parameter-efficient deep learning approach to predict conversion from mild cognitive impairment to alzheimer's disease. Neuroimage 189, 276-287 (2019)

44. Fisher, C.K., Smith, A.M., Walsh, J.R.: Machine learning for comprehensive forecasting of alzheimer's disease progression. Scientific reports 9(1), 1-14 (2019)

45. Liu, L., Zhao, S., Chen, H., Wang, A.: A new machine learning method for identifying alzheimer's disease. Simulation Modelling Practice and Theory 99, 102023 (2020)

46. Khan, A., Zubair, S.: An improved multi-modal based machine learning approach for the prognosis of alzheimer's disease. Journal of King Saud University-Computer and Information Sciences (2020)

47. Battineni, G., Chintalapudi, N., Amenta, F., Traini, E.: A comprehensive machine-learning model applied to magnetic resonance imaging (mri) to predict alzheimer's disease (ad) in older subjects. Journal of Clinical Medicine 9(7), 2146 (2020)

48. Ahmad, I., Pothuganti, K.: Analysis of different convolution neural network models to diagnose alzheimer's disease. Materials Today: Proceedings (2020)

49. Aghili, M., Tabarestani, S., Adjouadi, M., Adeli, E.: Predictive modeling of longitudinal data for alzheimer's disease diagnosis using rnns. In: International Workshop on PRedictive Intelligence In MEdicine, pp. 112-119 (2018). Springer

\section{Figures}

Figure 1 Self-Portraits of an artist with Alzheimer's disease.

Figure 2 Methodology workflow.

Figure 3 Data set summary.

Figure 4 Unique PTRACCAT values (only subjects with non missing data are considered). 
Figure 5 Linear correlation heat map for the data set.

Figure 6 Scatter plot between ADAS11 \& MMSE.

Figure 7 Comparison of multiple imputation algorithms performance.

Figure 8 Targets' distributions before data imputation.

Figure 9 Oversampling algorithms comparison.

Figure $10 \mathrm{~K}$-neighbours validation.

Figure 11 Targets' distributions after data balancing.

Figure 12 Confusion matrix for the Random Forest model.

Figure 13 Confusion matrix for the XGBoost model.

Figure 14 Random Forest model's variables importance.

Figure 15 Variables importance plot for $\mathrm{CN}$ diagnosis.

Figure 16 Variables importance plot for $\mathrm{LMCl}$ diagnosis.

Figure 17 Variables importance plot for AD diagnosis.

Figure 18 Dependence plot between WholeBrain and MidTemp for the LMCl class.

Figure 19 Dependence plot between CDRSB and MMSE for the AD class.

Figure 20 Dependence plot between FDG and CDRSB for the AD class.

Figure 21 Features influence on a subject with $\mathrm{CN}$ diagnosis to be predicted as $\mathrm{CN}$.

Figure 22 Features influence on a subject with CN diagnosis to be predicted as SMC.

Figure 23 Comparison between features impact on the predicted (AD) and true class ( $L M C I)$ of a subject. 
Figure 24 Comparison between features impact on the predicted $(\mathrm{EMCl})$ and true class $(\mathrm{SMC})$ of a subject.

Figure 25 Comparison between features impact on the predicted $(\mathrm{CN})$ and true class (SMC) of a subject.

Figure 26 Comparison between features impact on the predicted ( $\mathrm{LMCl})$ and true class $(A D)$ of a subject.

Figure 27 Summary variables importance plot for XGBoost model.

Figure 28 Variables importance plot for CN diagnosis.

Figure 29 Variables importance plot for $\mathrm{LMCl}$ diagnosis.

Figure 30 Variables importance plot for AD diagnosis

Figure 31 Dependence plots between FDG and CDRSB for different classes.

Figure 32 Dependence plot between APOE4 and AGE for AD class.

Figure 33 Dependence plot between RAVLT_immediate and MidTemp for LMCl class.

Figure 34 Features influence on a subject with AD diagnosis to be predicted as AD.

Figure 35 Features influence on a subject with $A D$ diagnosis to be predicted as $\mathrm{LMCl}$.

Figure 36 Comparison between features impact on the predicted (AD) and true class (LMCI) of a subject.

Figure 37 Comparison between features impact on the predicted $(\mathrm{EMCl})$ and true class $(\mathrm{SMC})$ of a subject.

Figure 38 Comparison between features impact on the predicted (CN) and true class (SMC) of a subject.

Figure 39 Comparison between features impact on the predicted $(\mathrm{LMCl})$ and true class $(A D)$ of a subject. 
Table 1 Random Forest and XGBoost classification performance for each class.

\begin{tabular}{rcccccccc} 
& \multicolumn{2}{c}{ Precision } & \multicolumn{2}{c}{ Recall } & \multicolumn{2}{c}{ Specificity } & \multicolumn{2}{c}{ F1 - score } \\
\hline & RF & XGB & RF & XGB & RF & XGB & RF & XGB \\
\hline CN & 0.81 & 0.86 & 0.84 & 0.90 & 0.95 & 0.96 & 0.83 & 0.88 \\
EMCI & 0.76 & 0.81 & 0.81 & 0.85 & 0.94 & 0.95 & 0.78 & 0.83 \\
LMCI & 0.69 & 0.78 & 0.75 & 0.81 & 0.91 & 0.94 & 0.72 & 0.80 \\
SMC & 0.90 & 0.94 & 0.72 & 0.78 & 0.98 & 0.99 & 0.80 & 0.85 \\
AD & 0.82 & 0.85 & 0.82 & 0.87 & 0.96 & 0.96 & 0.82 & 0.86 \\
\hline
\end{tabular}

Table 2 Comparison of models' performance.

\begin{tabular}{cccccccc}
\hline & Precision & Recall & Specificity & F1-score & Accuracy & Training time & Prediction time \\
\hline XGBoost Classifier & 0.85 & 0.79 & 0.96 & 0.84 & 0.842 & $20.8 \mathrm{~s}$ & $103 \mathrm{~ms}$ \\
Random Forest Classifier & 0.78 & 0.79 & 0.95 & 0.79 & 0.787 & $5.16 \mathrm{~s}$ & $157 \mathrm{~ms}$ \\
\hline
\end{tabular}

Table 3 Comparison of our model with some of the contestants of TADPOLE Challenge [42]

\begin{tabular}{|c|c|c|c|c|c|c|c|}
\hline & Feature selection & Features & $\begin{array}{l}\text { Missing data } \\
\text { imputation }\end{array}$ & Prediction model & BCA & Training time & $\begin{array}{l}\text { Prediction time } \\
\text { (per subject) }\end{array}$ \\
\hline Frog & Automatic & 490 & None & Gradient boosting & 0.849 & $1 \mathrm{~h}$ & \\
\hline Our XGB Model & Manual & 13 & $\begin{array}{l}\text { Extra Trees Re- } \\
\text { gressor }\end{array}$ & XGBoost & 0.842 & $20.8 \mathrm{sec}$ & $0.03 \mathrm{~ms}$ \\
\hline BenchmarkSVM & Manual & 6 & $\begin{array}{l}\text { Mean of previ- } \\
\text { ous values }\end{array}$ & SVM & 0.764 & $20 \mathrm{sec}$ & $0.001 \mathrm{sec}$ \\
\hline $\begin{array}{l}\text { SMALLHEADS } \\
\text { - NeuralNet }\end{array}$ & Automatic & 376 & $\begin{array}{l}\text { Nearest neigh- } \\
\text { bour }\end{array}$ & Deep NN & 0.605 & $40 \mathrm{~min}$ & $0.06 \mathrm{sec}$ \\
\hline Rocket & Manual & 6 & $\begin{array}{l}\text { Median of diag- } \\
\text { nostic group }\end{array}$ & $\begin{array}{l}\text { Linear mixed effects } \\
\text { model }\end{array}$ & 0.519 & $5 \mathrm{~min}$ & $0.3 \mathrm{sec}$ \\
\hline
\end{tabular}


Table 4 Analysis of the latest eminent literature.

\begin{tabular}{|c|c|c|c|c|c|c|c|c|}
\hline \multirow{2}{*}{ Reference } & \multicolumn{5}{|c|}{ Dataset characteristics } & \multirow{2}{*}{ Methodology } & \multirow{2}{*}{ Results } & \multirow{2}{*}{ Comment } \\
\hline & Size & Features & Origin & Description & Split & & & \\
\hline [44] & $\begin{array}{l}1909 \\
\text { subjects } \\
\text { (MCl or } \\
\text { AD) }\end{array}$ & 44 & $\begin{array}{l}\text { Coalition } \\
\text { Against } \\
\text { Major } \\
\text { Dis- } \\
\text { eases } \\
\text { (CAMD) }\end{array}$ & $\begin{array}{l}\text { ADAS-Cog and } \\
\text { MMSE scores, } \\
\text { laboratory and } \\
\text { clinical tests, } \\
\text { background } \\
\text { information }\end{array}$ & $\begin{array}{l}\text { Train: } \\
75 \% \\
\text { Vali- } \\
\text { date: } \\
5 \% \\
\text { Test: } \\
20 \%\end{array}$ & $\begin{array}{l}\text { Conditional } \\
\text { Restricted } \\
\text { Boltzmann Ma- } \\
\text { chine (CRBM) }\end{array}$ & $\begin{array}{l}\text { Accuracy: } 0.5 \\
\text { (differentiation } \\
\text { between actual } \\
\text { and synthetic } \\
\text { patient data) } \\
\text { R2: } 0.82 \pm 0.01 \\
\text { (observed } \\
\text { vs. predicted } \\
\text { correlation ) }\end{array}$ & $\begin{array}{l}\text { Synthetic trajectories starting } \\
\text { for real patients and entirely } \\
\text { synthetic patients are gener- } \\
\text { ated. Missing data imputation } \\
\text { is performed. CRBM does not } \\
\text { model correlation between cog- } \\
\text { nitive scores and other variables } \\
\text { very well. Some crucial param- } \\
\text { eters, such as levels of amy- } \\
\text { loid, are omitted from the } \\
\text { dataset. Overall performances of } \\
\text { the model are significant. }\end{array}$ \\
\hline [45] & $\begin{array}{l}36 \text { sub- } \\
\text { jects } \\
\text { (HC: } \\
13, \mathrm{AD}: \\
23) \text { : } \\
32 \text { (HC: } \\
8, \quad A D: \\
\text { 24) }\end{array}$ & $\begin{array}{l}504 \\
488\end{array}$ & $\begin{array}{l}\text { VBSD / } \\
\text { Dem@Care }\end{array}$ & $\begin{array}{l}\text { Extracted } \\
\text { espectrogram } \\
\text { features from } \\
\text { subjects' voices. } \\
\text { Each recording } \\
\text { is previously } \\
\text { segmented. }\end{array}$ & $\begin{array}{l}\text { Train: } \\
\text { 35/31Test } \\
\text { 1(sub- } \\
\text { jects) }\end{array}$ & $\begin{array}{l}\text { Logistic Regres- } \\
\text { : sion CV (best } \\
\text { among others) }\end{array}$ & 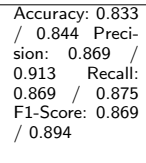 & $\begin{array}{l}\text { It provides new and inventive ap- } \\
\text { proach for analyzing and predict- } \\
\text { ing the disease. No data prepro- } \\
\text { cessing is performed. Even after } \\
\text { the segmentation, datasets are } \\
\text { still small-sized. Hyperparameter } \\
\text { tuning is not applied. }\end{array}$ \\
\hline [46] & $\begin{array}{l}343 \\
\text { sessions } \\
-150 \\
\text { subjects } \\
\text { (ND: } \\
72 \\
\text { D: } 78)\end{array}$ & 15 & $\begin{array}{l}\text { Open } \\
\text { Access } \\
\text { Se- } \\
\text { ries of } \\
\text { Imaging } \\
\text { Studies } \\
\text { (OA- } \\
\text { SIS) }\end{array}$ & $\begin{array}{l}\text { MRI scans and } \\
\text { other brain } \\
\text { measurements, } \\
\text { MMSE and } \\
\text { CDR scores, } \\
\text { demographic } \\
\text { data }\end{array}$ & $\begin{array}{l}\text { Random } \\
\text { selec- } \\
\text { tion } \\
\text { alloca- } \\
\text { tion for } \\
\text { train, } \\
\text { validate } \\
\text { and test }\end{array}$ & $\begin{array}{lr}\text { Random } & \text { Forest } \\
\text { (best } & \text { among } \\
\text { others) } & \end{array}$ & $\begin{array}{lr}\text { Accuracy: } & 0.868 \\
\text { Precision: } & 0.941 \\
\text { Recall: } & 0.8 \\
\text { AUC: } & 0.872\end{array}$ & $\begin{array}{l}\text { Detailed data processing and } \\
\text { examination. Complete work- } \\
\text { flow following consecutive } \\
\text { stages from data preprocessing } \\
\text { to model evaluation. Only } \\
\text { first visit for each patient is } \\
\text { taken into account (e.g. cases } \\
\text { when a patient convert from } \\
\text { non-demented to demented are } \\
\text { omitted). Only simple imputing } \\
\text { techniques are considered. }\end{array}$ \\
\hline [47] & $\begin{array}{l}373 \\
\text { sessions } \\
-\quad 150 \\
\text { subjects } \\
(\mathrm{ND}: 72, \\
\mathrm{D}: 64 \\
\mathrm{C}: 14)\end{array}$ & 15 & $\begin{array}{l}\text { Open } \\
\text { Access } \\
\text { Se- } \\
\text { ries of } \\
\text { Imaging } \\
\text { Studies } \\
\text { (OA- } \\
\text { SIS) }\end{array}$ & $\begin{array}{l}\text { MRI scans and } \\
\text { other brain } \\
\text { measurements, } \\
\text { MMSE and } \\
\text { CDR scores, } \\
\text { demographic } \\
\text { data }\end{array}$ & $\begin{array}{l}10 \text {-fold } \\
\text { cross- } \\
\text { validation }\end{array}$ & $\begin{array}{l}\text { Hybrid mod- } \\
\text { eling (combi- } \\
\text { nation of four } \\
\text { models) }\end{array}$ & $\begin{array}{l}\text { Accuracy: } 0.980 \\
\text { Precision: } 0.981 \\
\text { Recall: } \quad 0.980 \\
\text { ROC: } 0.991\end{array}$ & $\begin{array}{l}\text { Three different approaches are } \\
\text { being analyzed: manual feature } \\
\text { selection, automatic feature se- } \\
\text { lection and hybrid modeling. Re- } \\
\text { sults obtained by hybrid mod- } \\
\text { eling are fascinating, contain- } \\
\text { ing high and stabile values. Not } \\
\text { a single stage of data prepro- } \\
\text { cessing and engineering is per- } \\
\text { formed. }\end{array}$ \\
\hline [48] & $\begin{array}{l}5000 \\
\text { images } \\
\text { (Mild, } \\
\text { Very } \\
\text { Mild, } \\
\text { Non, } \\
\text { Moder- } \\
\text { ate De- } \\
\text { mented) }\end{array}$ & $\begin{array}{l}1700 \\
\text { region } \\
\text { propos- } \\
\text { als per } \\
\text { image }\end{array}$ & $\begin{array}{l}\text { Alzheimer' } \\
\text { Disease } \\
\text { Neu- } \\
\text { roimag- } \\
\text { ing } \\
\text { Initia- } \\
\text { tive } \\
\text { (ADNI) }\end{array}$ & $\begin{array}{l}\text { 'sMRI scan im- } \\
\text { ages }\end{array}$ & $\begin{array}{l}\text { Separate } \\
\text { datasets } \\
\text { for train } \\
\text { and test }\end{array}$ & 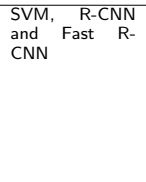 & 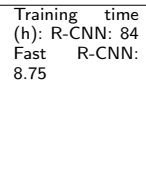 & $\begin{array}{l}\text { The main goal is to provide com- } \\
\text { parison between different object } \\
\text { detection algorithms in terms } \\
\text { of their training and predicting } \\
\text { times. No prediction results and } \\
\text { accuracy metrics are shown. No } \\
\text { data preprocessing is shown. }\end{array}$ \\
\hline [49] & $\begin{array}{l}1721 \\
\text { subjects } \\
(521 \\
\mathrm{NC}, \\
864 \\
\mathrm{MCl} \\
336 \\
\mathrm{AD})\end{array}$ & 47 & $\begin{array}{l}\mathrm{AD} \\
\text { Neu- } \\
\text { roimag- } \\
\text { ing } \\
\text { Initia- } \\
\text { tive } \\
\text { (ADNI) }\end{array}$ & $\begin{array}{l}\text { MRI and PET } \\
\text { scans, CSF, } \\
\text { gene expression } \\
\text { and cognitive } \\
\text { scores }\end{array}$ & $\begin{array}{l}\text { Train: } \\
70 \\
\% \text { Val- } \\
\text { idate: } \\
115 \% \\
\text { Test: } \\
15 \%\end{array}$ & $\begin{array}{l}\text { Recurrent Neu- } \\
\text { ral Network }\end{array}$ & $\begin{array}{l}\text { Accuracy:- AD - } \\
\text { NC: } 0.959 \cdot A D- \\
\text { MCl: } 0.859 \text { NC - } \\
\text { MCl: } 0.773\end{array}$ & $\begin{array}{l}\text { Whole focus is put on the RNN } \\
\text { algorithm its possibilities and } \\
\text { its evaluation. Filling data be- } \\
\text { tween different timestamps is } \\
\text { performed on three various ap- } \\
\text { proaches. No information about } \\
\text { data preprocessing is given. No } \\
\text { missing data imputation is per- } \\
\text { formed (missing values are re- } \\
\text { placed with } 0 \text { ). }\end{array}$ \\
\hline [11] & $\begin{array}{l}202 \\
\text { subjects } \\
(52 \mathrm{HC}, \\
99 \mathrm{MCl} \\
51 \mathrm{AD})\end{array}$ & $\begin{array}{l}189 \\
\text { (MRI } \\
\text { ROI: } \\
93, \\
\text { PET } \\
\text { ROI: } \\
\text { 93, } \\
\text { CSF: 3) }\end{array}$ & $\begin{array}{l}\text { Alzheimer' } \\
\text { Disease } \\
\text { Neu- } \\
\text { roimag- } \\
\text { ing } \\
\text { Initia- } \\
\text { tive } \\
\text { (ADNI) }\end{array}$ & $\begin{array}{l}\text { 'sMRI, FDG- } \\
\text { PET and CSF } \\
\text { biomarkers }\end{array}$ & $\begin{array}{l}10 \text {-fold } \\
\text { cross- } \\
\text { validation }\end{array}$ & $\begin{array}{l}\text { SVM (multiple } \\
\text { kernel combina- } \\
\text { tion) }\end{array}$ & $\begin{array}{l}\text { Accuracy: } 0.932 \\
\text { Specificity: } \\
0.933 \text { Recall: } \\
0.930 \quad\end{array}$ & $\begin{array}{l}\text { This study represents unified } \\
\text { way of combining data from dif- } \\
\text { ferent sources into one kernel. } \\
\text { Only three types of data are be- } \\
\text { ing used. An improvement of one } \\
\text { model's effectiveness using pre- } \\
\text { cise feature selection is shown. } \\
\text { Before usage, images are prepro- } \\
\text { cessed. }\end{array}$ \\
\hline [10] & $\begin{array}{l}\text { Group I: } \\
\text { CN:20, } \\
\text { AD:20;Gro } \\
\text { II: } \\
\text { CN: } 14, A D \\
\text { III: CN: } \\
\text { 57, } \\
\text { AD: } 33 ; \\
\text { Group } \\
\text { IV: } \\
\text { FTLD: } \\
\text { 19 }\end{array}$ & $\begin{array}{l}- \\
\text { oup } \\
\text { :14; Group }\end{array}$ & $\begin{array}{l}\text { Each } \\
\text { group } \\
\text { of sub- } \\
\text { jects } \\
\text { comes } \\
\text { from } \\
\text { dif- } \\
\text { ferent } \\
\text { com- } \\
\text { munity } \\
\text { or re- } \\
\text { search } \\
\text { center }\end{array}$ & MR scans & $\begin{array}{l}\text { Leave- } \\
\text { one-out } \\
\text { tech- } \\
\text { nique }\end{array}$ & SVM & $\begin{array}{l}\text { Group I / Group } \\
\text { II / Group III } \\
\text { / Group IV: Ac- } \\
\text { curacy: } 0.950 / / \\
0.929 / 0.811 \\
\text { / } 0.892 \text { Speci- } \\
\text { ficity: } 0.950 / \\
0.857 / 0.930 \\
/ 0.947 \text { Recall: } \\
0.950 / 1.00 / \\
0.606 / 0.833\end{array}$ & $\begin{array}{l}\text { Differentiation between AD and } \\
\text { FTLD subjects is represented } \\
\text { as they are often misidentified. } \\
\text { Detailed image preprocessing is } \\
\text { performed. Results are better } \\
\text { than most of the scientific works } \\
\text { that used MRI before. Only two } \\
\text { diagnoses at a time are taken } \\
\text { into classification. }\end{array}$ \\
\hline [43] & $\begin{array}{l}785 \\
\text { subjects } \\
(\quad 184 \\
\mathrm{HC}, \\
228 \\
\text { sMCl, } \\
181 \\
\text { pMCl, } \\
192 \\
\text { AD) }\end{array}$ & - & $\begin{array}{l}\mathrm{AD} \\
\text { Neu- } \\
\text { roimag- } \\
\text { ing } \\
\text { Initia- } \\
\text { tive } \\
\text { (ADNI) }\end{array}$ & $\begin{array}{l}\text { ROI, APOe4, } \\
\text { cognitive scores } \\
\text { and demo- } \\
\text { graphic data }\end{array}$ & $\begin{array}{l}10 \text {-fold } \\
\text { cross- } \\
\text { validation }\end{array}$ & CNN & $\begin{array}{l}\text { Accuracy: } 0.925 \\
\text { Specificity: } \\
0.850 \quad \text { Recall: } \\
0.875\end{array}$ & $\begin{array}{l}\text { Very detailed and mathemati- } \\
\text { cally supported approach of us- } \\
\text { ing neural networks for clas- } \\
\text { sification is presented. Data } \\
\text { preprocessing and feature se- } \\
\text { lection is performed. Special } \\
\text { attention is put on avoiding } \\
\text { over/underfitting problems. All } \\
\text { data is baseline. }\end{array}$ \\
\hline
\end{tabular}

Table 5 Random Forest global interpretability.

\begin{tabular}{|c|c|c|c|c|c|c|c|c|c|c|c|c|c|c|c|c|c|c|c|c|c|c|c|c|}
\hline \multirow{2}{*}{ Diagnosis } & \multirow{2}{*}{\multicolumn{3}{|c|}{\begin{tabular}{|l|l|}
\multicolumn{2}{|c|}{ Demographics } \\
Gender & Age \\
\end{tabular}}} & \multirow{2}{*}{\multicolumn{2}{|c|}{ Education }} & \multirow{2}{*}{\multicolumn{2}{|c|}{$\begin{array}{l}\text { CDRSB } \\
\text { Low High } \\
\end{array}$}} & \multirow{2}{*}{\multicolumn{2}{|c|}{\begin{tabular}{c}
\multicolumn{2}{c}{ Cognitive scc } \\
MMSE \\
\end{tabular}}} & \multirow{2}{*}{\multicolumn{2}{|c|}{$\begin{array}{l}\text { res } \\
\text { RAVL__immediate } \\
\text { Low High } \\
\end{array}$}} & \multirow{2}{*}{\multicolumn{4}{|c|}{\begin{tabular}{|l|r|} 
WholeBrain & Mippocampus \\
\end{tabular}}} & \multirow{2}{*}{\multicolumn{4}{|c|}{ 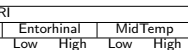 }} & \multirow{2}{*}{\multicolumn{2}{|c|}{\begin{tabular}{c|c} 
& PET \\
FDG \\
Low High \\
\end{tabular}}} & \multirow{2}{*}{\multicolumn{3}{|c|}{\begin{tabular}{|c|} 
Genotype \\
APOE 4 \\
\end{tabular}}} \\
\hline & & & & & & & & & & & & & & & & & & & & & & & & \\
\hline $\begin{array}{l}\mathrm{CN} \\
\mathrm{EMCl}\end{array}$ & + & $\bar{i}$ & $x$ & $\begin{array}{l}x \\
x\end{array}$ & ${ }_{x}^{+}$ & + & - & - & + & : & $+\frac{+}{x}$ & + & $i$ & $x$ & $\begin{array}{l}x \\
+\end{array}$ & $x$ & $\begin{array}{c}x \\
\end{array}$ & $x$ & $-\mathrm{s}$ & $:$ & $\begin{array}{r}x \\
\end{array}$ & $+\frac{+}{x}$ & & $x$ \\
\hline LMCI & + & + & $x$ & $\hat{-}$ & $\hat{+}$ & & $\hat{-}$ & 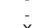 & $x$ & + & $\hat{-}$ & $\dot{x}$ & - & + & - & + & t & + & 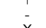 & + & 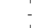 & & $x$ & + \\
\hline $\begin{array}{l}S M C \\
A D\end{array}$ & $\begin{array}{l}+ \\
+\end{array}$ & $\bar{x}$ & $\begin{array}{l}x \\
+\end{array}$ & 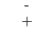 & - & + & i & $\begin{array}{l}x \\
+\end{array}$ & + & 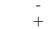 & + & : & $\stackrel{+}{+}$ & 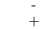 & + & $\begin{array}{l}x \\
x\end{array}$ & & $\begin{array}{l}x \\
x\end{array}$ & $\begin{array}{l}x \\
x\end{array}$ & 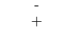 & + & $x$ & $\begin{array}{l}x \\
+\end{array}$ & $i$ \\
\hline
\end{tabular}


Table 6 XGBoost global interpretability.

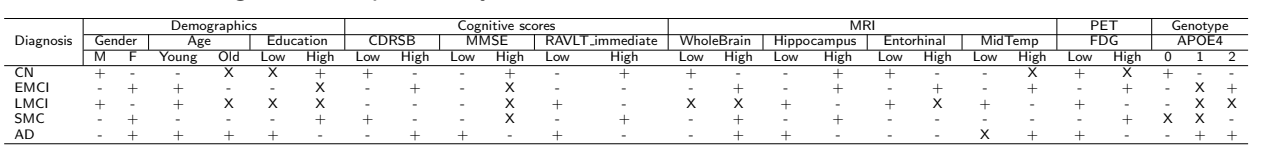

Table 7 Merged scheme showing the influence of each feature on each of the diagnosis.

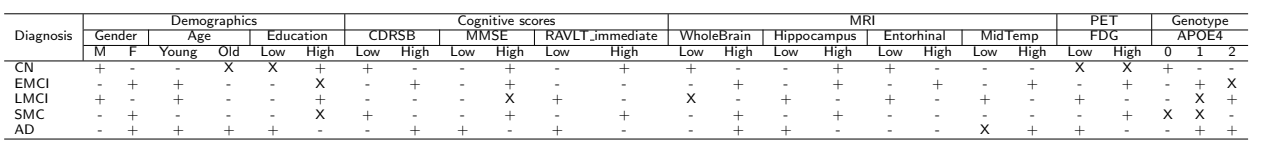


Figures

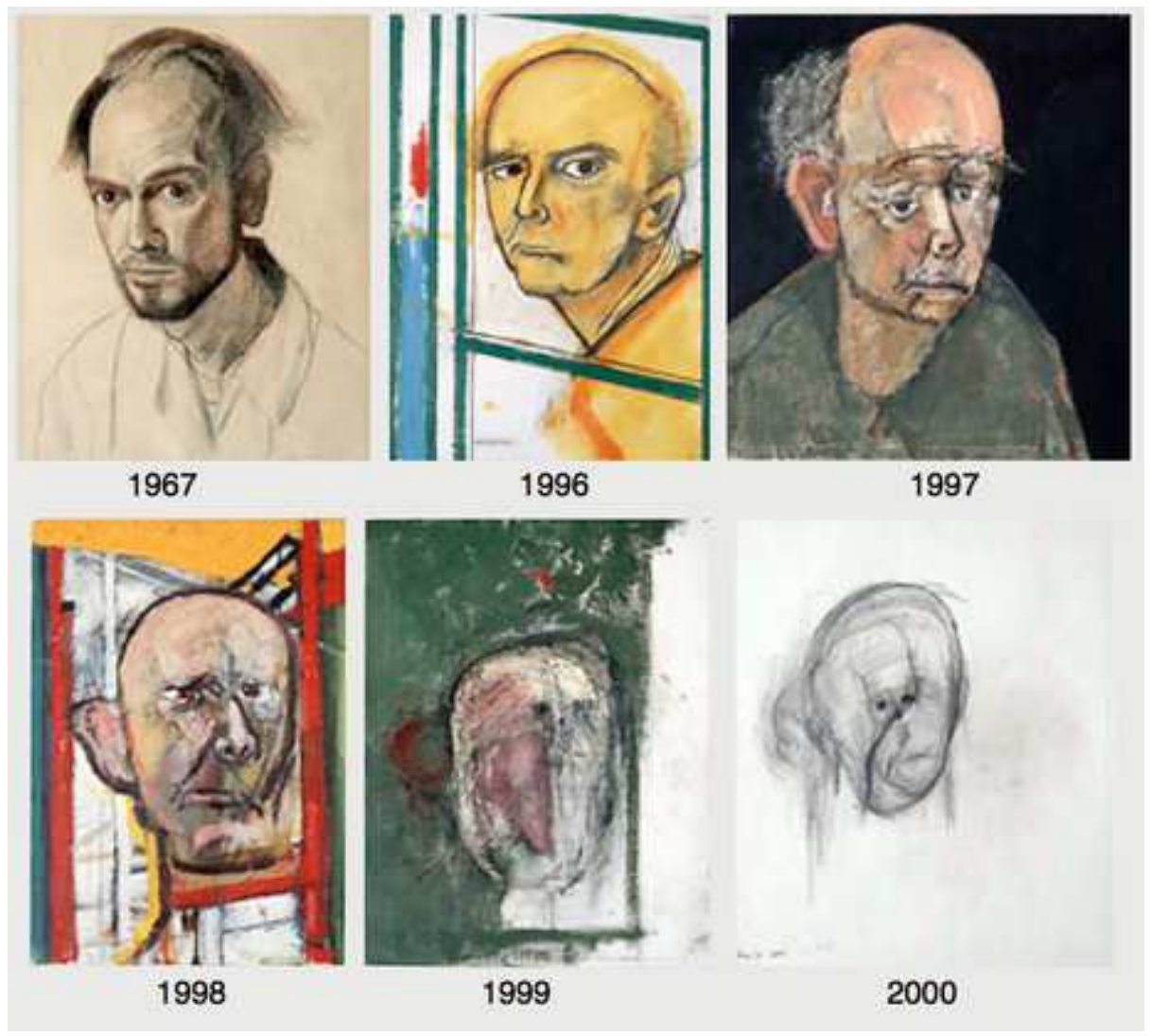

Figure 1

Self-Portraits of an artist with Alzheimer's disease. 


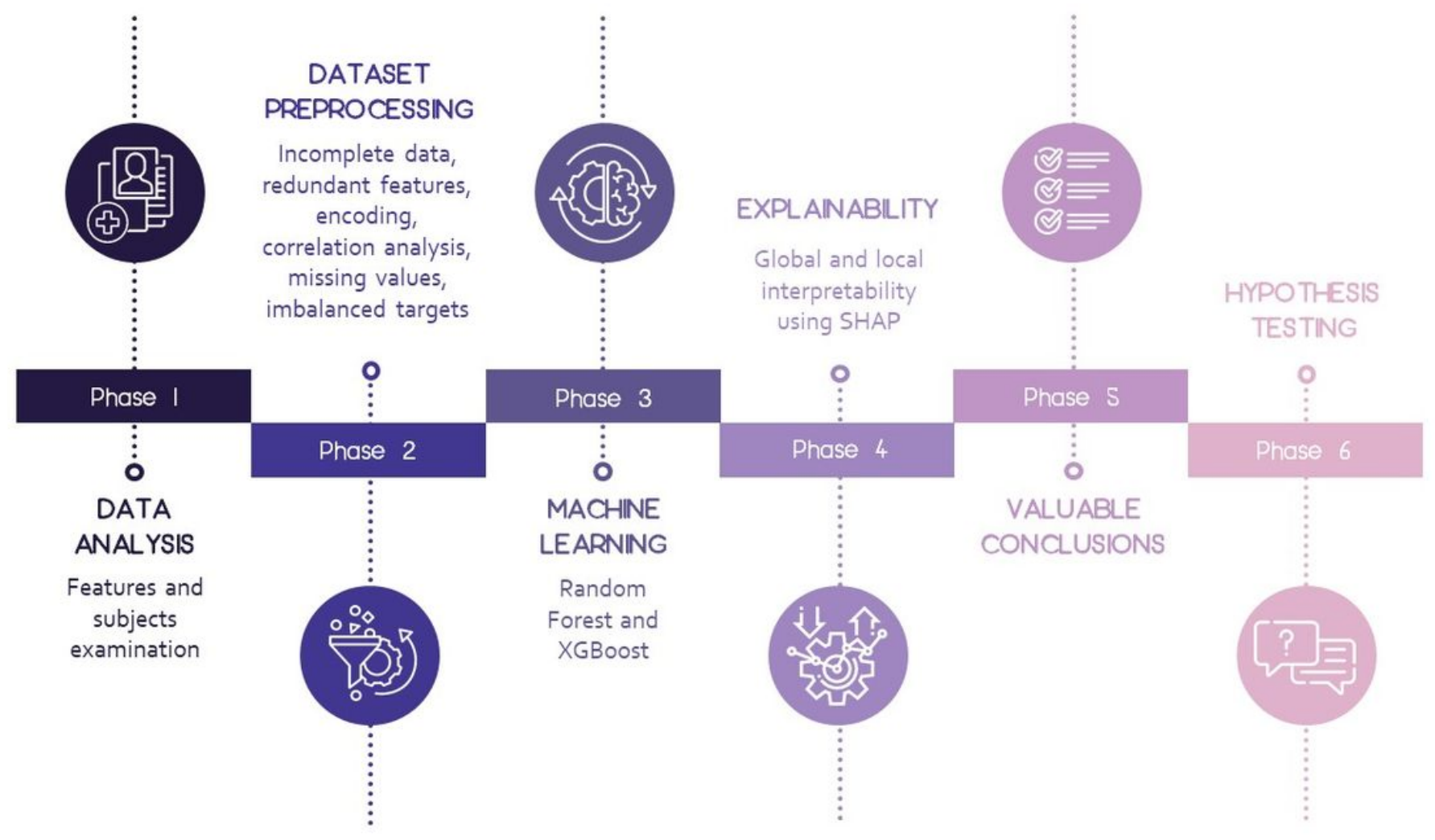

Figure 2

Methodology work ow.

RangeIndex: 12741 entries, 0 to 12740

Data columns (total 17 columns):

$\begin{array}{ll}\text { PTID } & 12741 \text { non-null object } \\ \text { AGE } & 12741 \text { non-null float64 } \\ \text { PTGENDER } & 12741 \text { non-null object } \\ \text { PTEDUCAT } & 12741 \text { non-null int64 } \\ \text { PTRACCAT } & 12741 \text { non-null object } \\ \text { APOE4 } & 12729 \text { non-null float64 } \\ \text { FDG } & 3352 \text { non-null float64 } \\ \text { AV45 } & 2118 \text { non-null float64 } \\ \text { CDRSB } & 8963 \text { non-null float64 } \\ \text { ADAS11 } & 8910 \text { non-null float64 } \\ \text { MMSE } & 8932 \text { non-null float64 } \\ \text { RAVLT_immediate } & 8831 \text { non-null float64 } \\ \text { Hippocampus } & 6802 \text { non-null float64 } \\ \text { WholeBrain } & 7689 \text { non-null float64 } \\ \text { Entorhinal } & 6469 \text { non-null float64 } \\ \text { MidTemp } & 6469 \text { non-null float64 } \\ \text { DX_bl } & 12741 \text { non-null object }\end{array}$

Figure 3 
Data set summary.

White

1046

Black

More than one 16

Asian

15

Unknown

Hawaiian/other PI

3

Am Indian/Alaskan

2

\section{Figure 4}

Unique PTRACCAT values (only subjects with non missing data are considered).

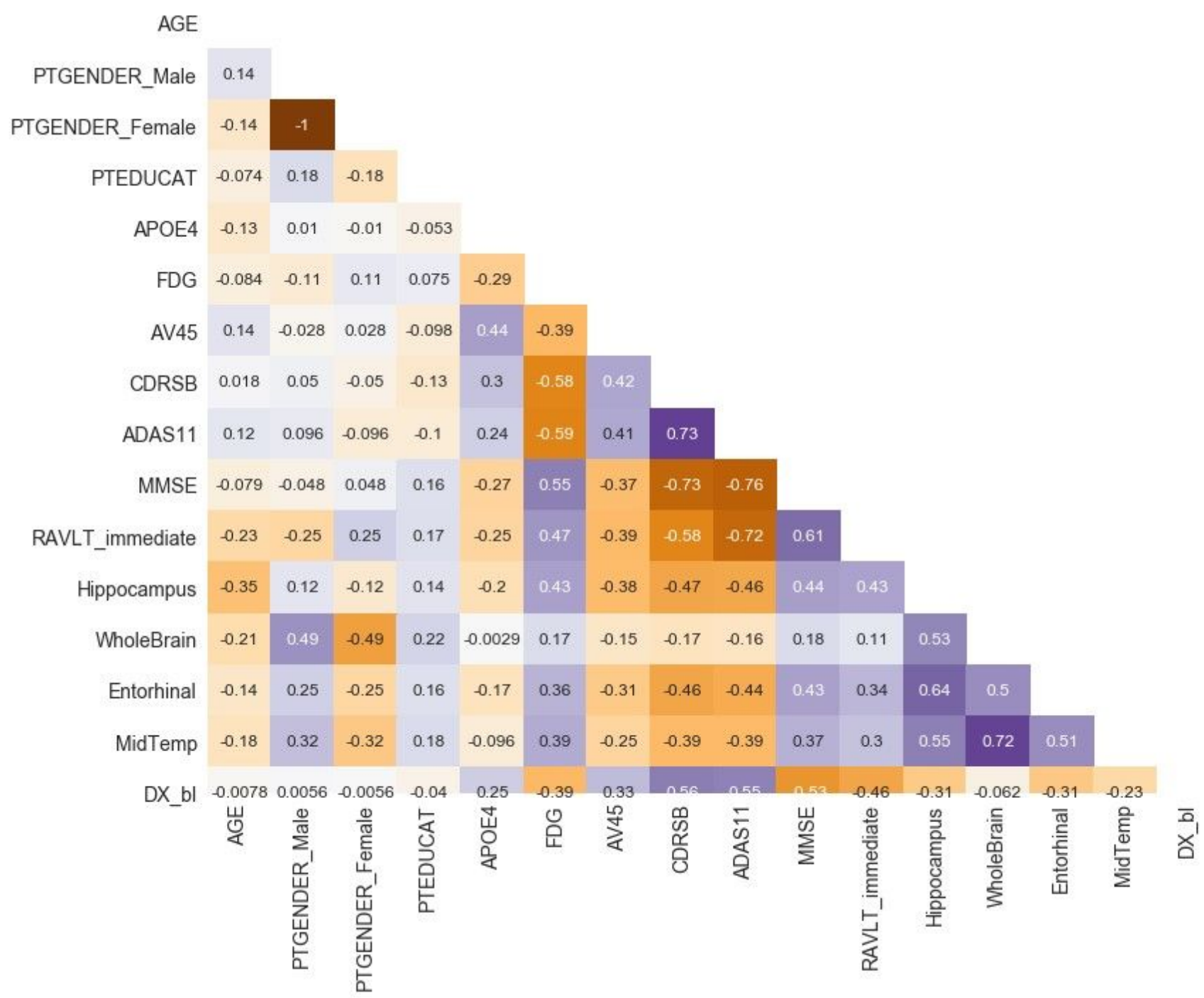

Figure 5

Linear correlation heat map for the data set. 


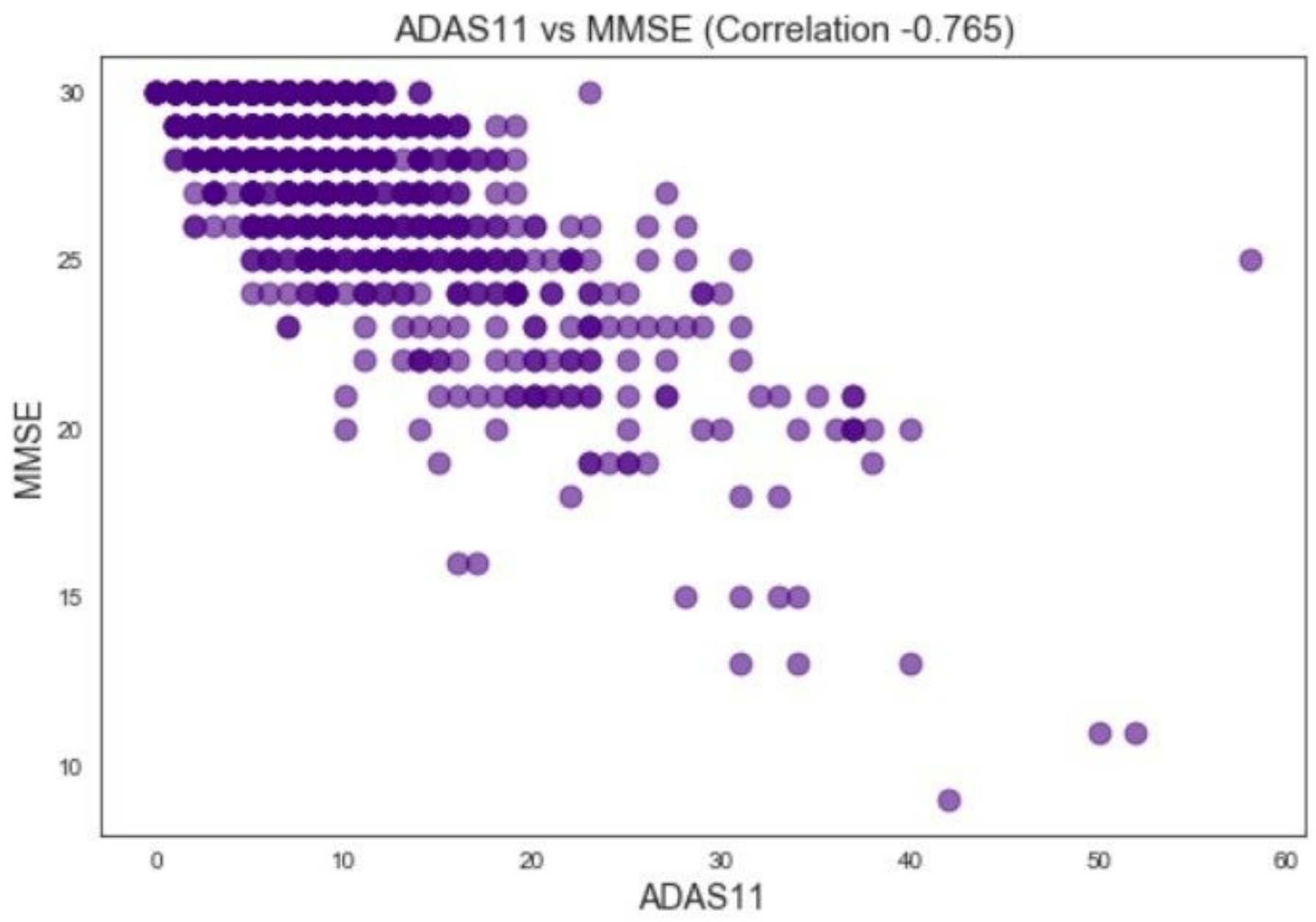

Figure 6

Scatter plot between ADAS11 \& MMSE. 


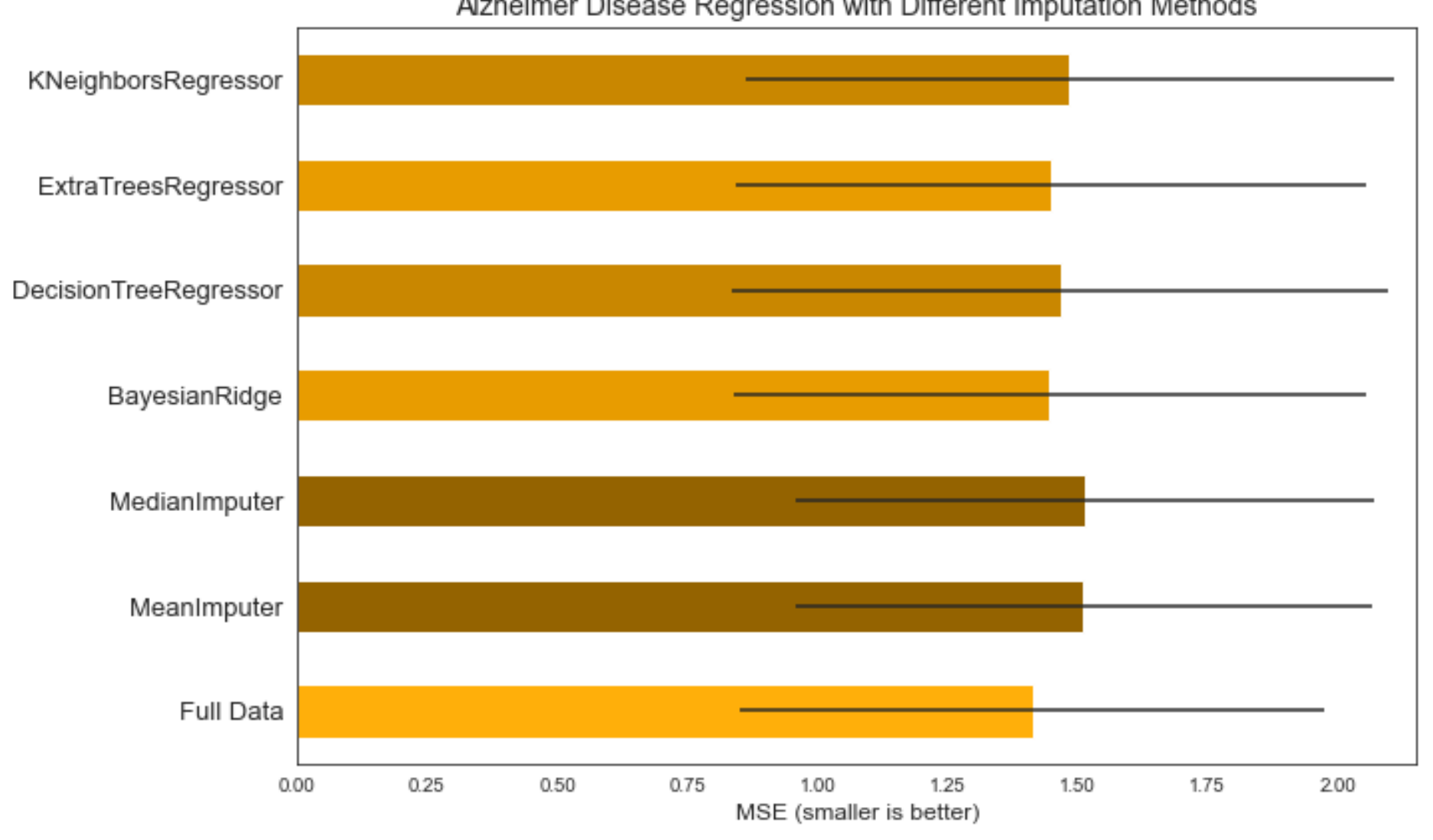

Figure 7

Comparison of multiple imputation algorithms performance.
Diagnosis
$\mathrm{DX} \mathrm{bl}$
Values

$\begin{array}{rcc}\text { LMCI } & 3.0 & 3526 \\ \text { CN } & 1.0 & 2652 \\ \text { EMCI } & 2.0 & 1854 \\ \text { AD } & 5.0 & 1196 \\ \text { SMC } & 4.0 & 364\end{array}$

\section{Figure 8}

Targets' distributions before data imputation. 


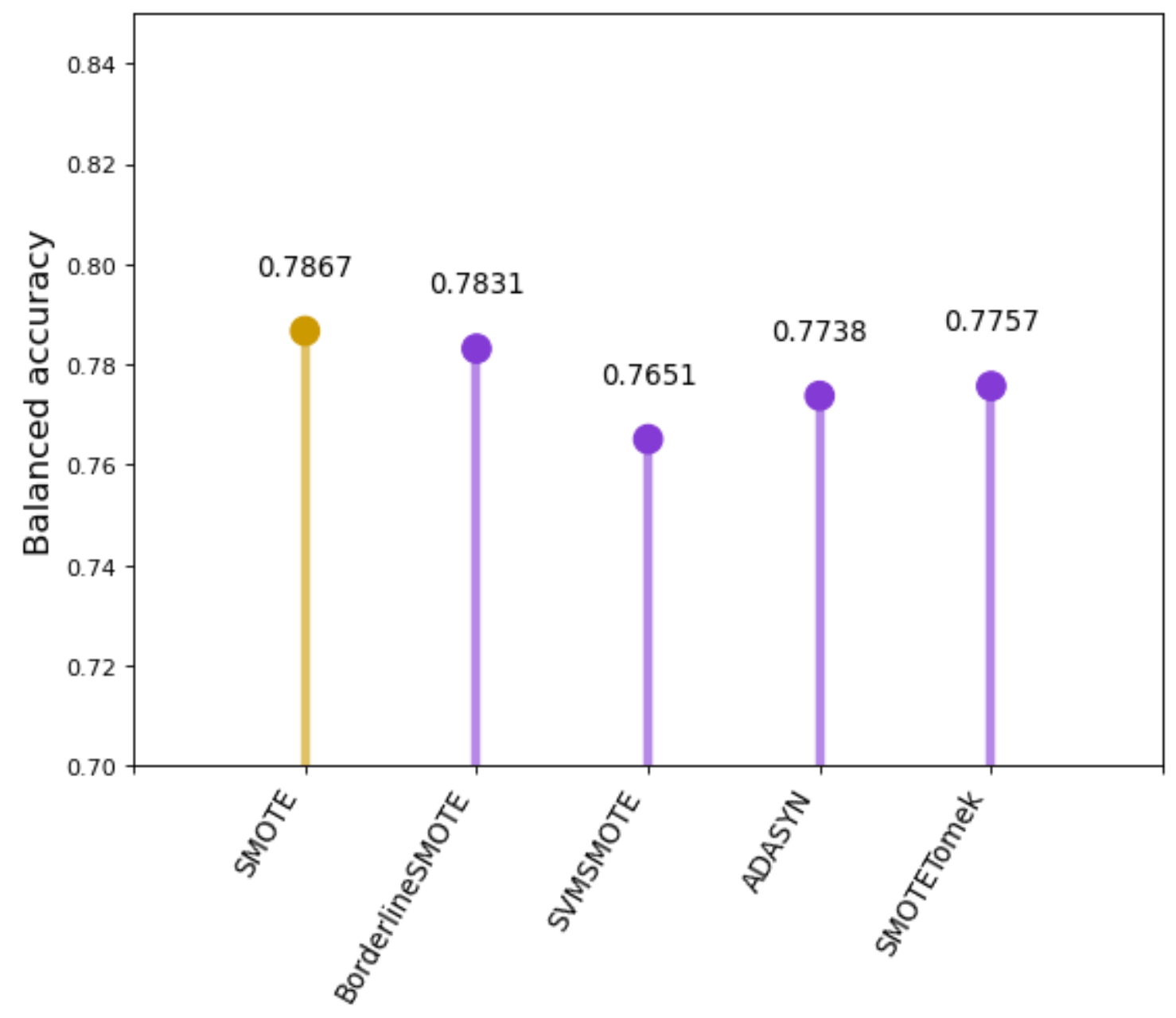

Figure 9

Oversampling algorithms comparison.

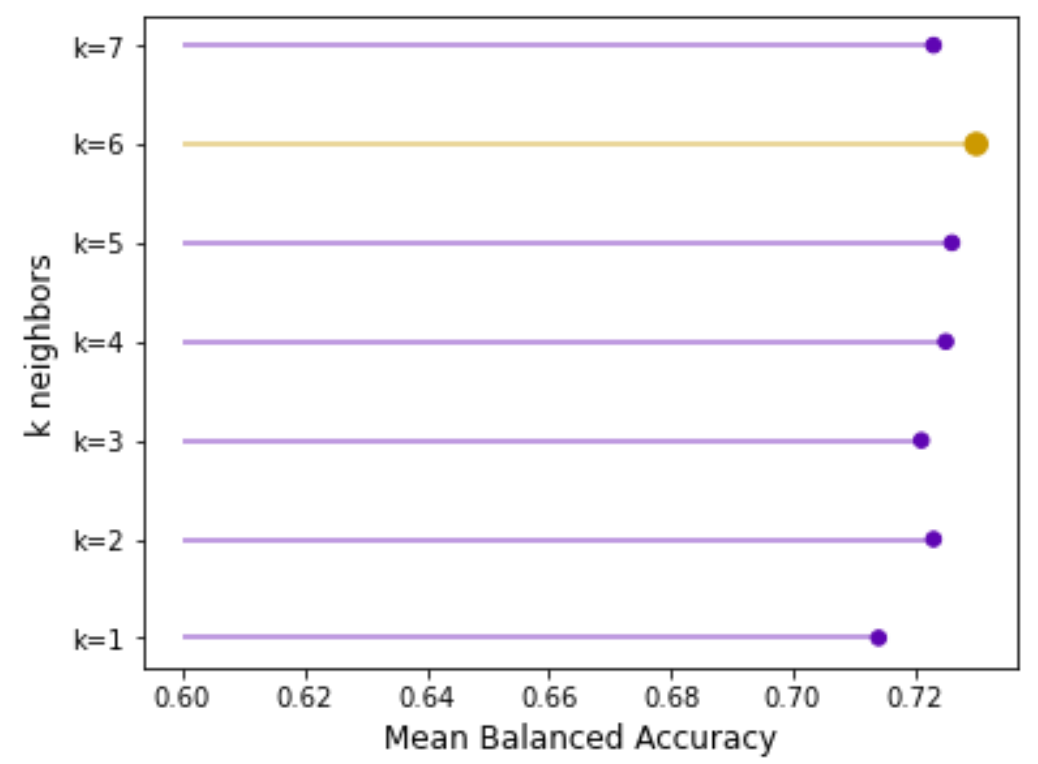

Figure 10 
K-neighbours validation.

Training Dataset Target Distribution

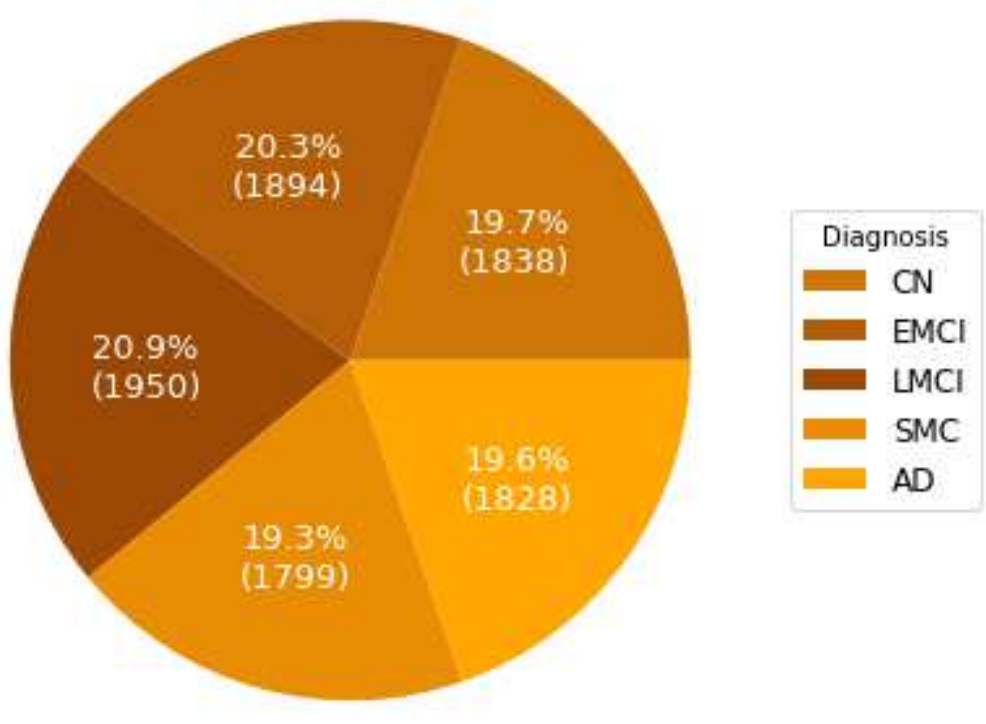

Testing Dataset Target Distribution

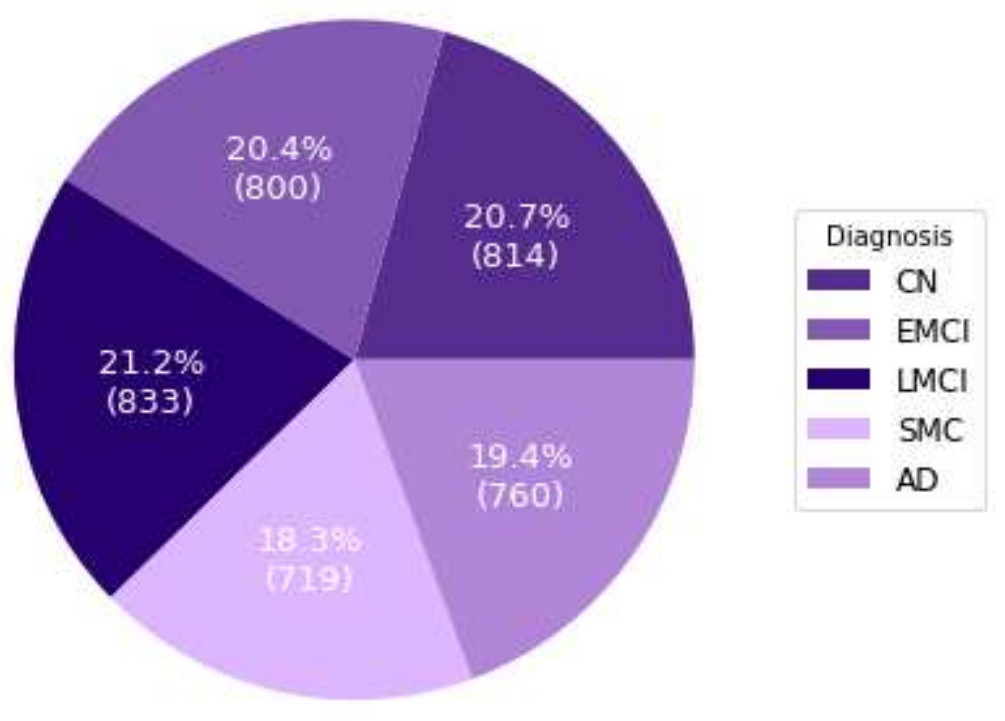

Figure 11

Targets' distributions after data balancing. 


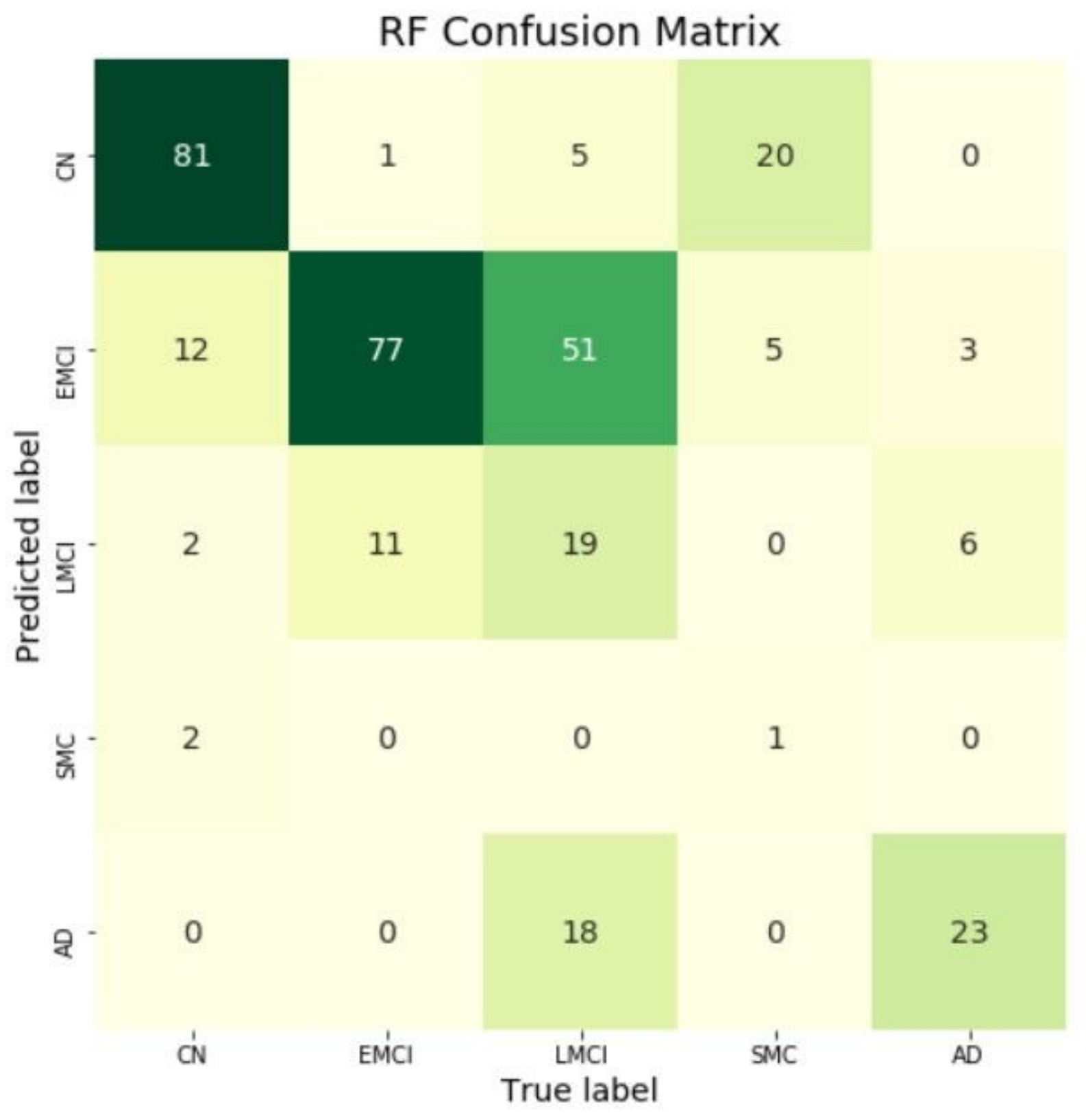

Figure 12

Confusion matrix for the Random Forest model. 


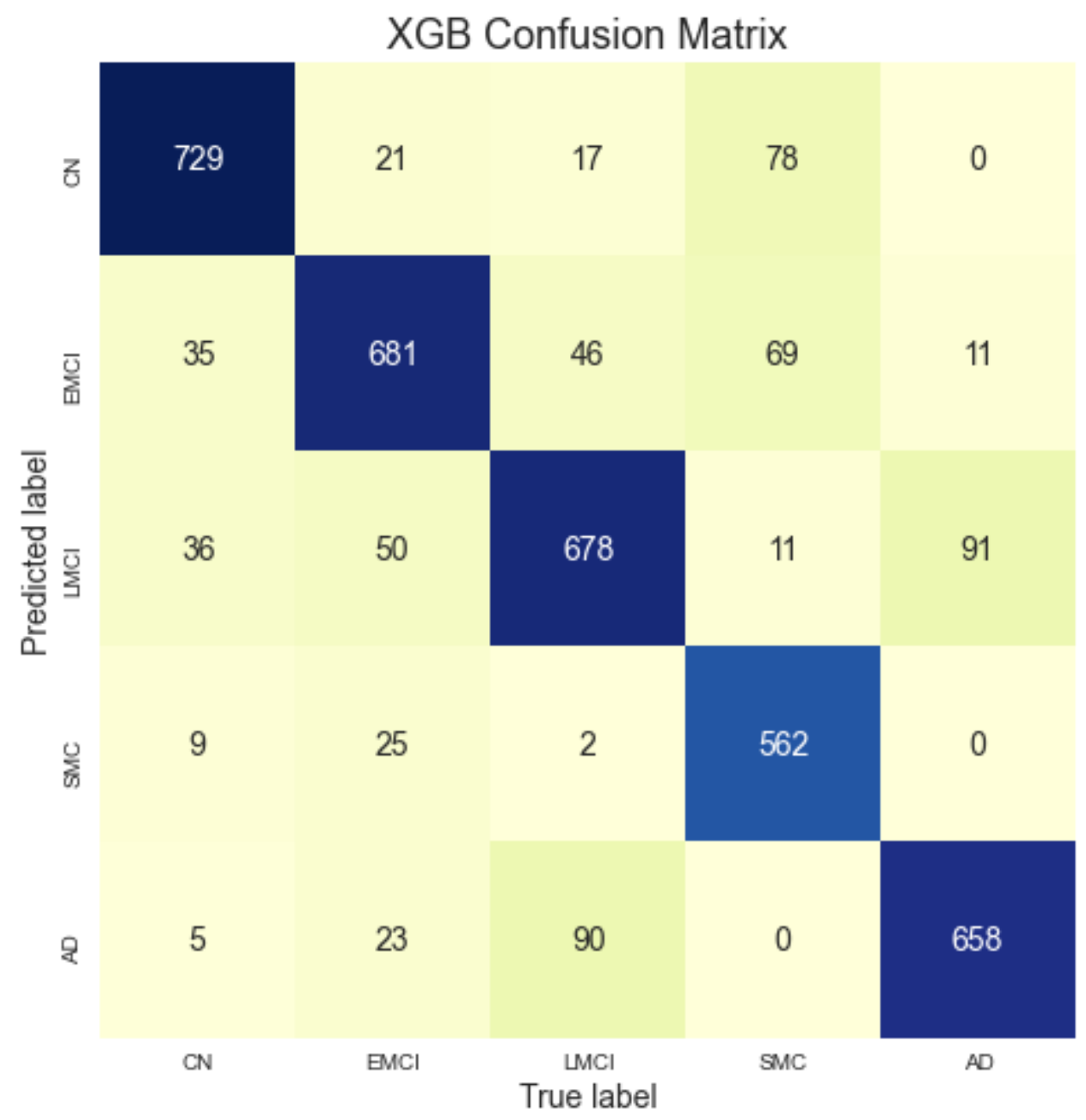

Figure 13

Confusion matrix for the XGBoost model. 


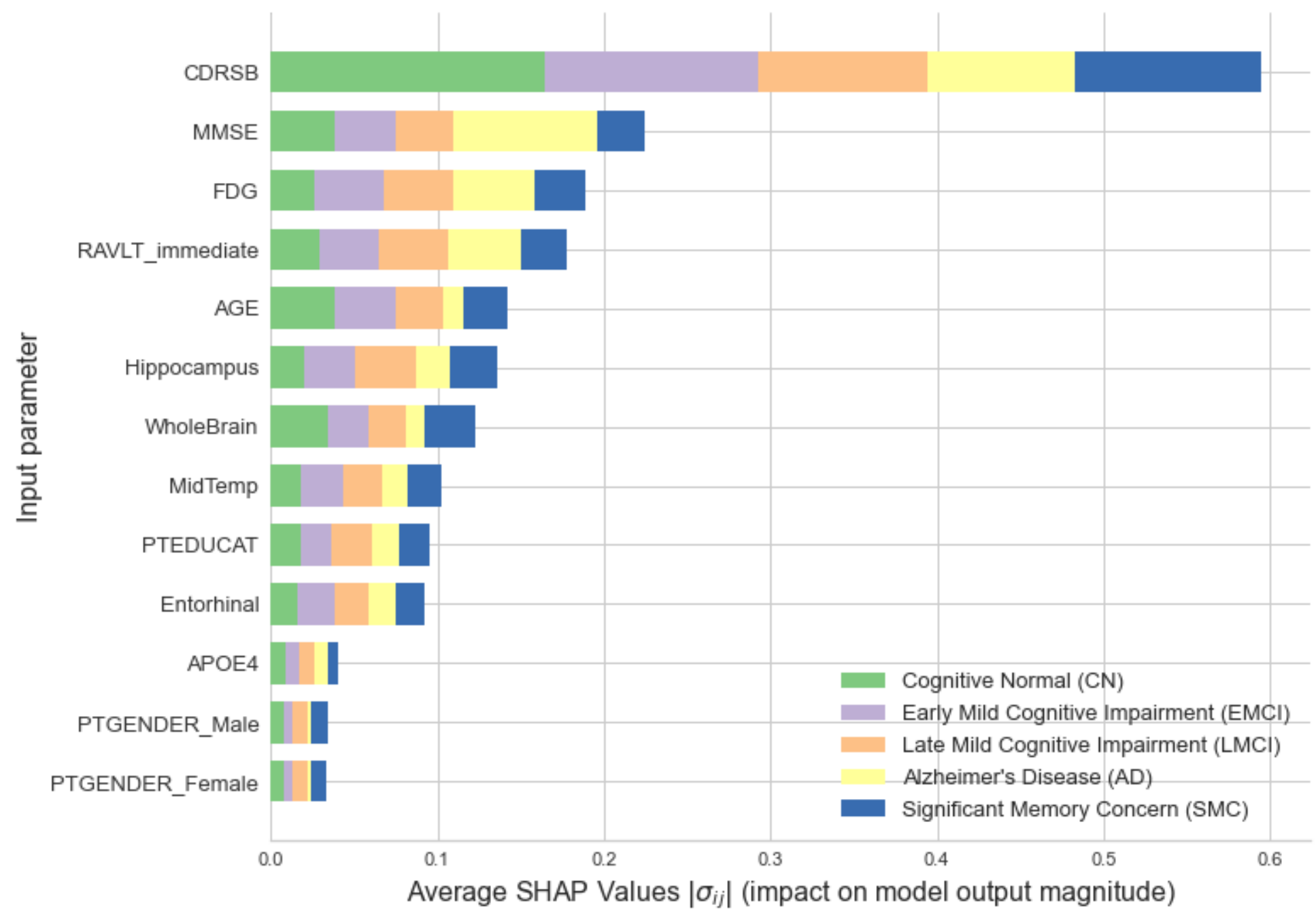

Figure 14

Random Forest model's variables importance. 


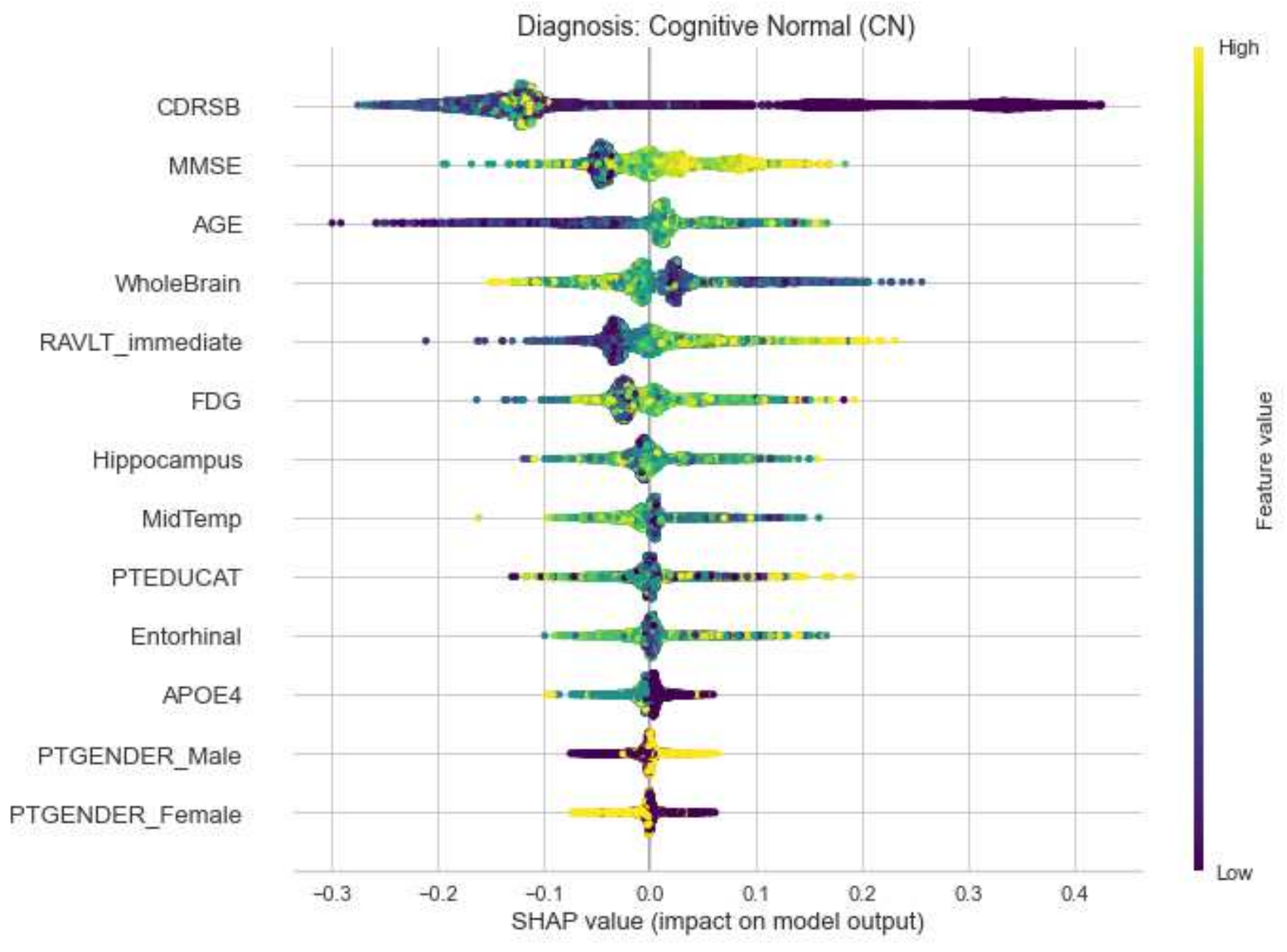

Figure 15

Variables importance plot for CN diagnosis. 
Diagnosis: Late Mild Cognitive Impairment (LMCl)

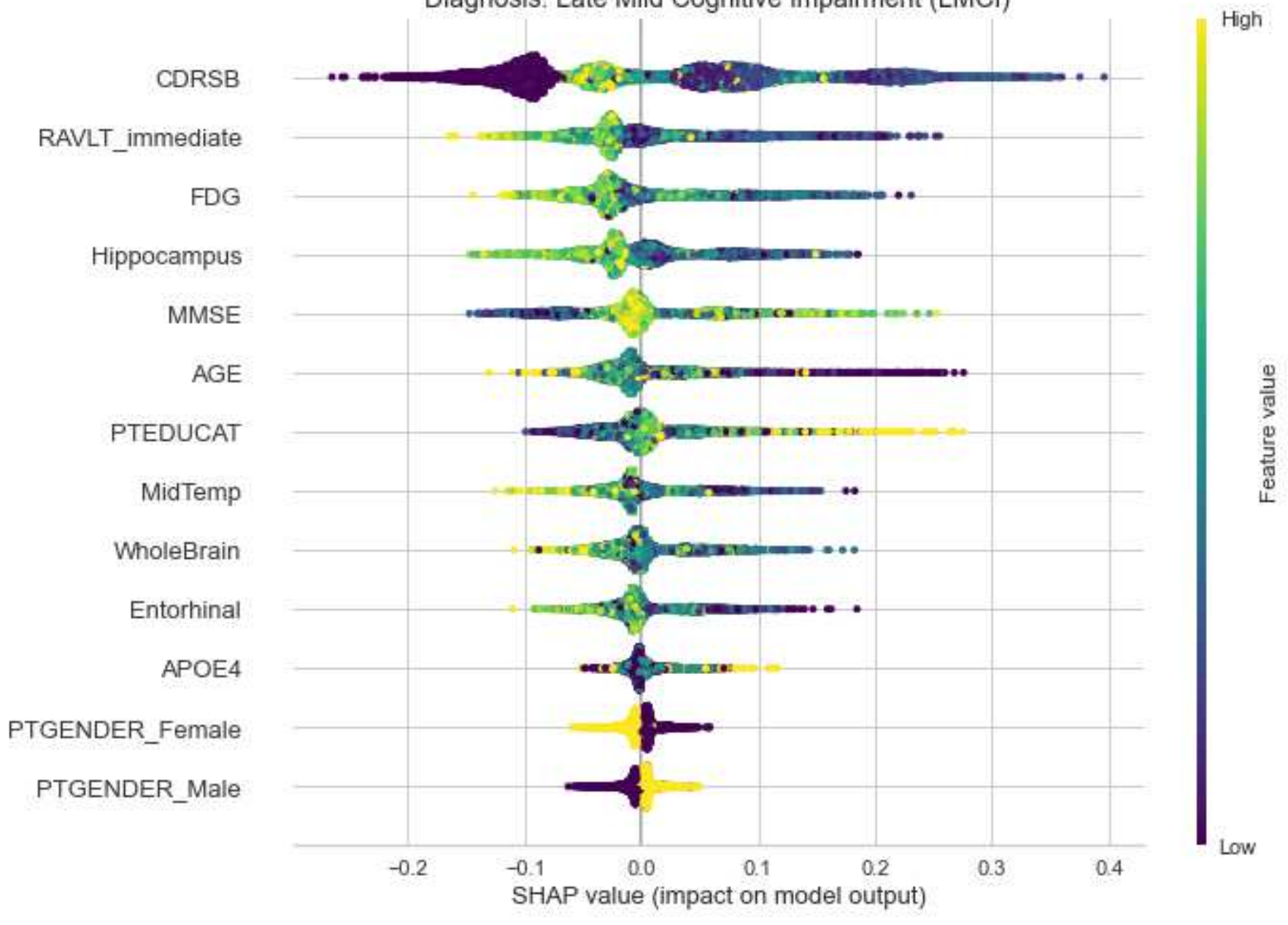

\section{Figure 16}

Variables importance plot for $\mathrm{LMCl}$ diagnosis. 
Diagnosis: Alzheimer's Disease (AD)

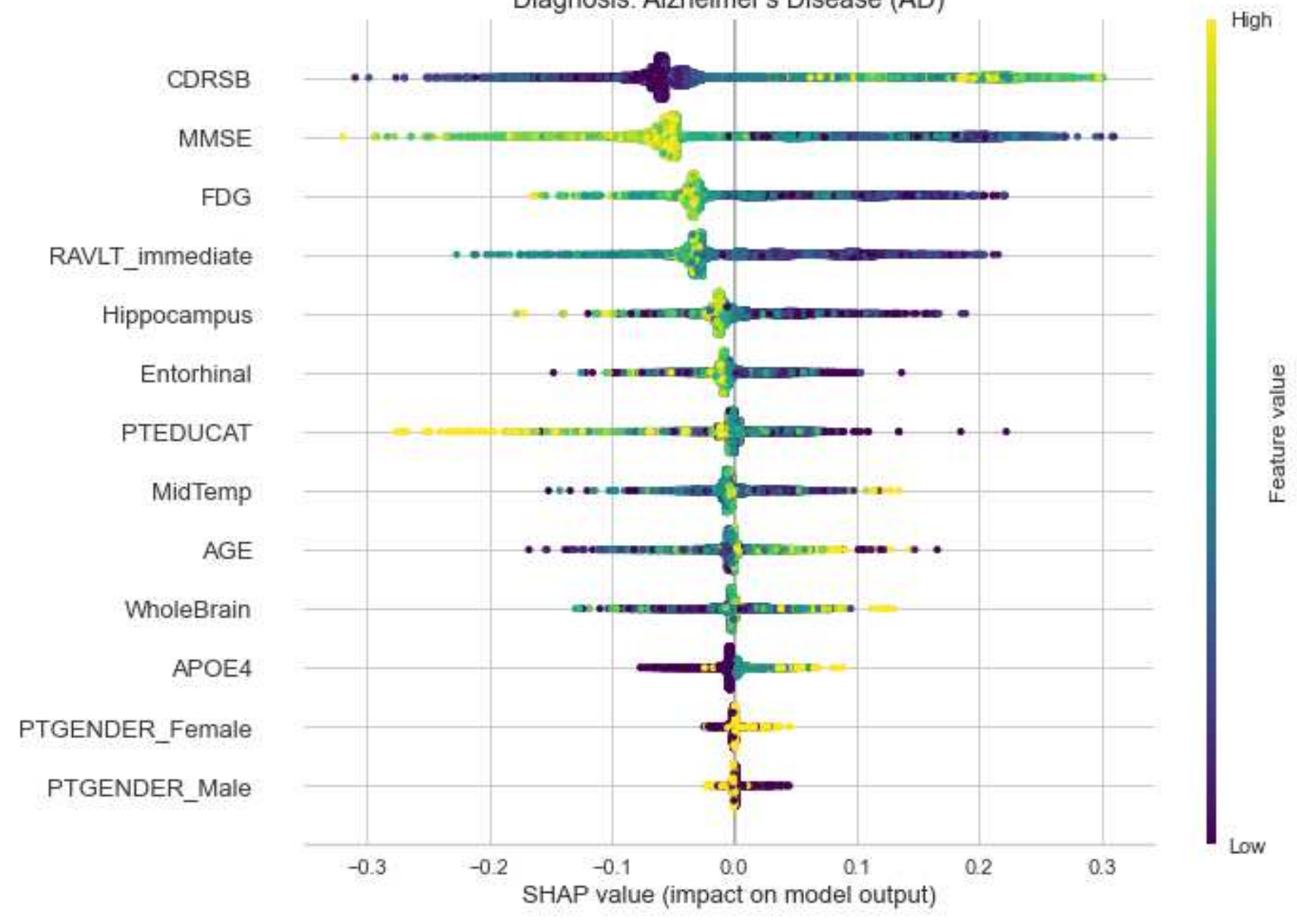

\section{Figure 17}

Variables importance plot for AD diagnosis.

Diagnosis: Late Mild Cognitive Impairment (LMCl)

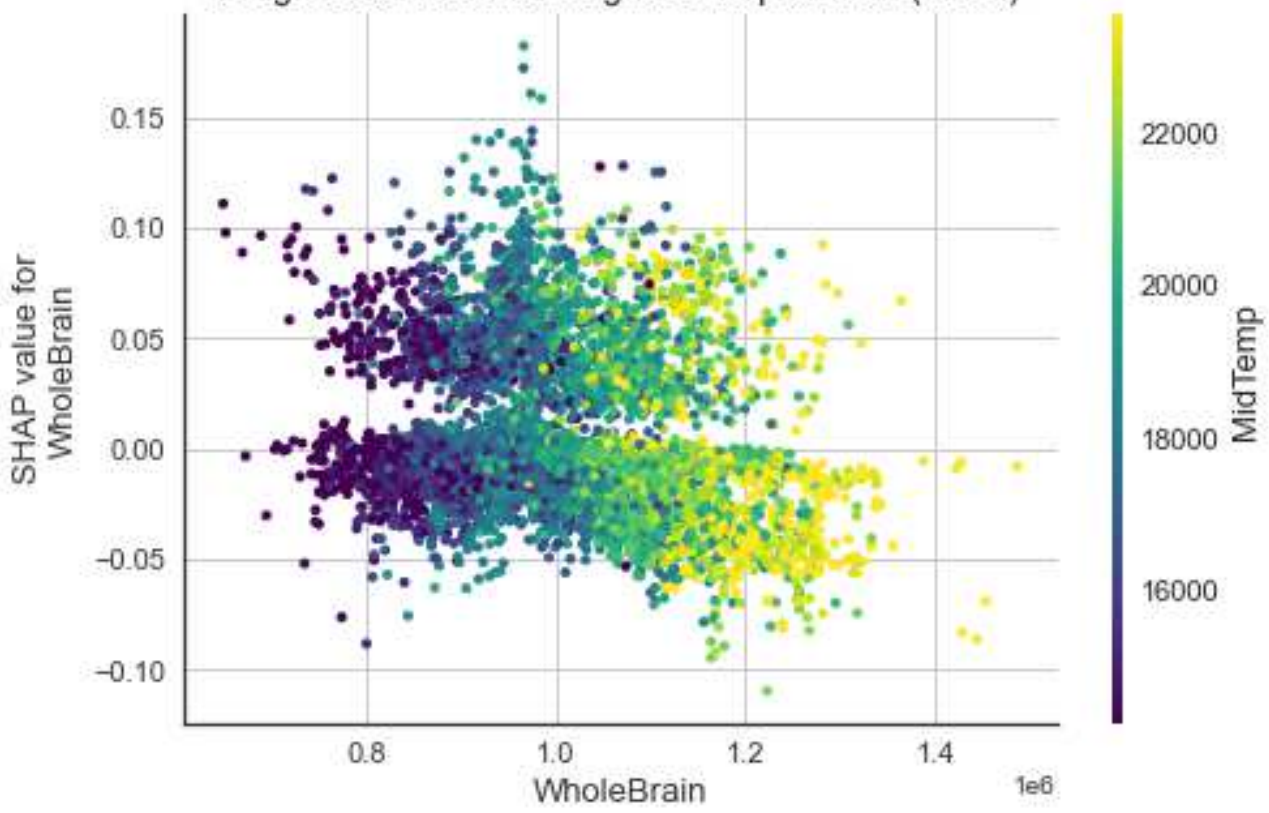


Figure 18

Dependence plot between WholeBrain and MidTemp for the LMCl class.

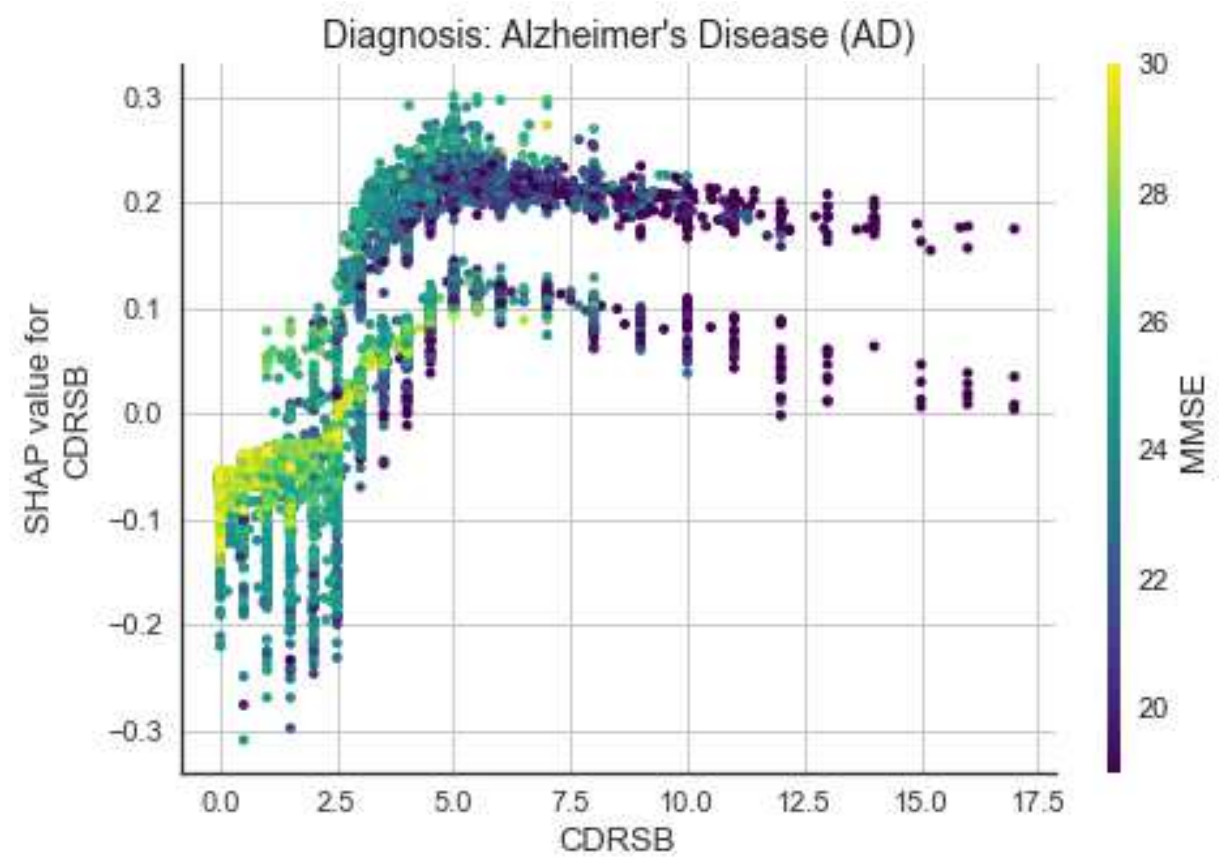

Figure 19

Dependence plot between CDRSB and MMSE for the AD class.

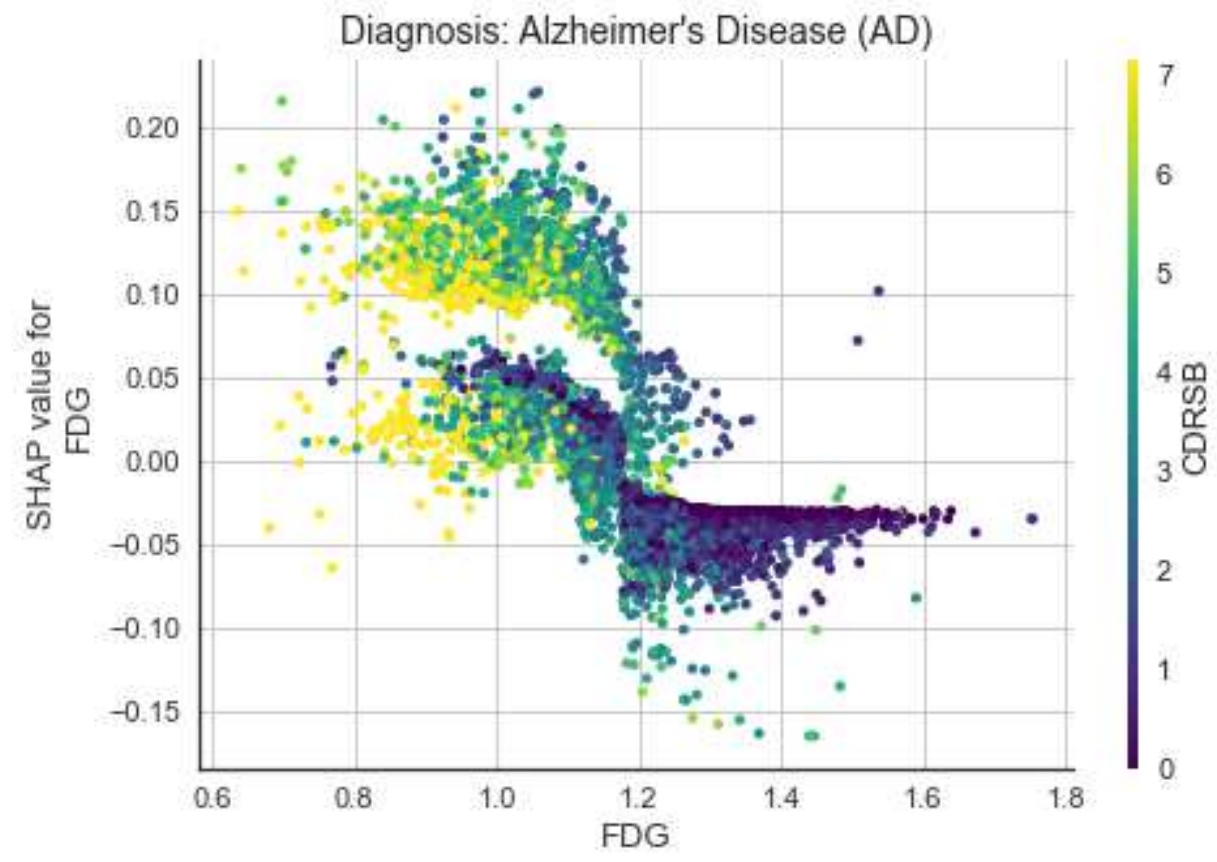

Figure 20

Dependence plot between FDG and CDRSB for the AD class. 


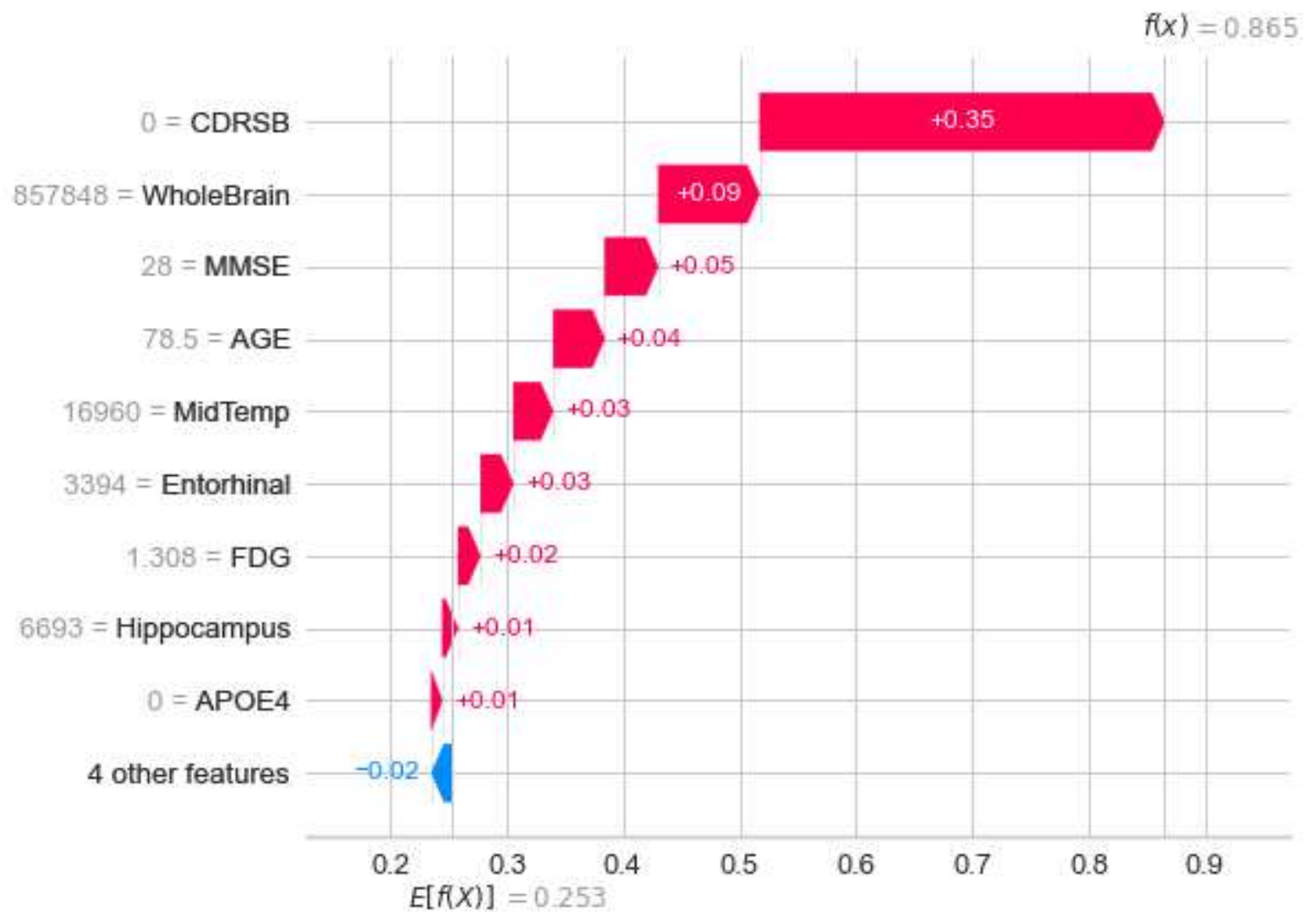

Figure 21

Features influence on a subject with $\mathrm{CN}$ diagnosis to be predicted as $\mathrm{CN}$.

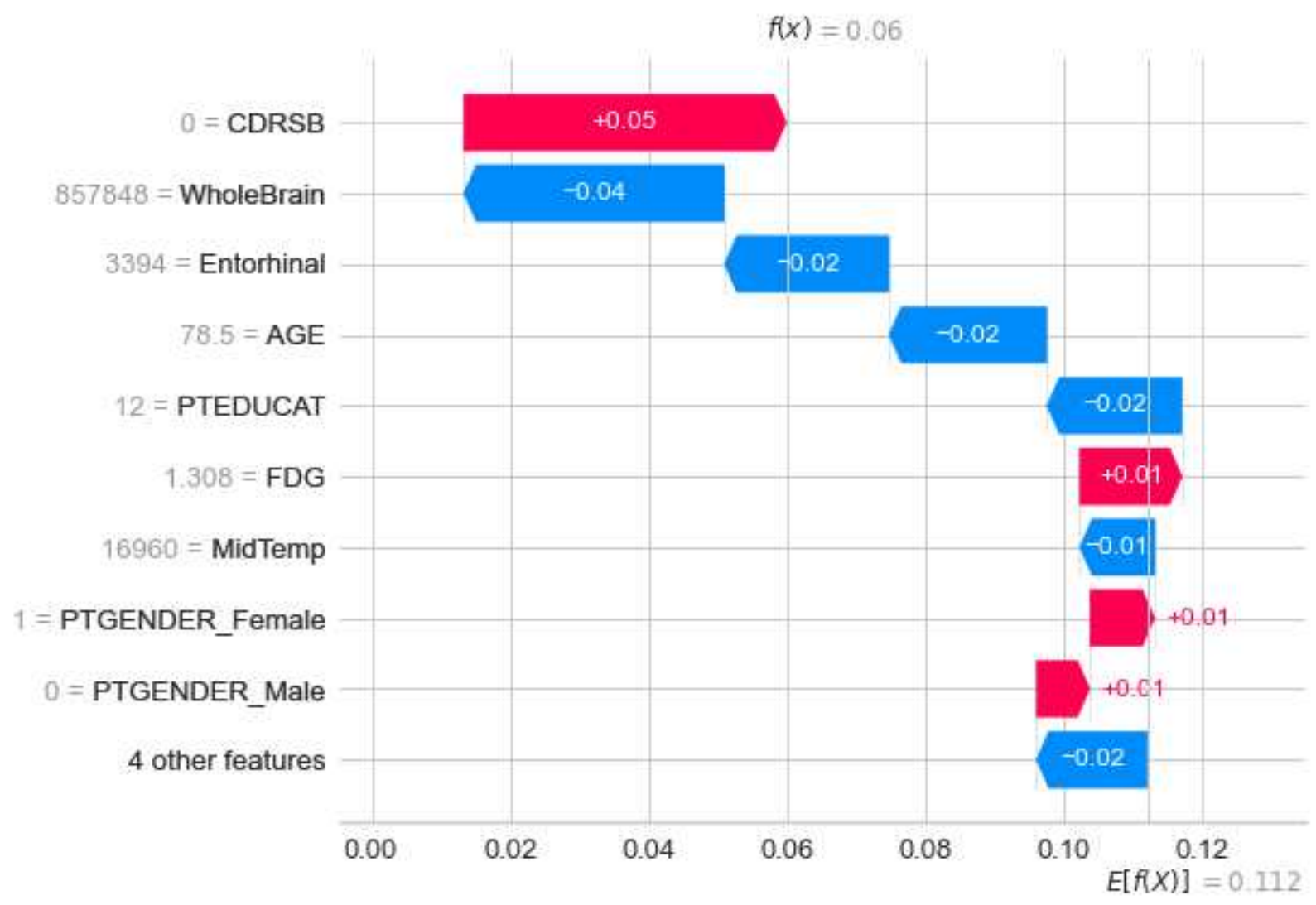


Figure 22

Features influence on a subject with $\mathrm{CN}$ diagnosis to be predicted as SMC.

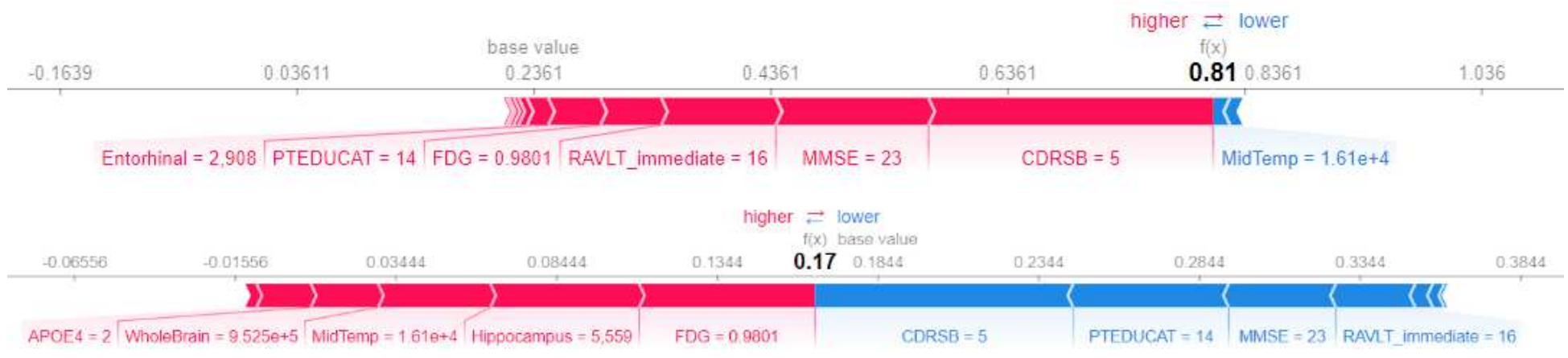

\section{Figure 23}

Comparison between features impact on the predicted (AD) and true class ( $\mathrm{LMCl})$ of a subject.

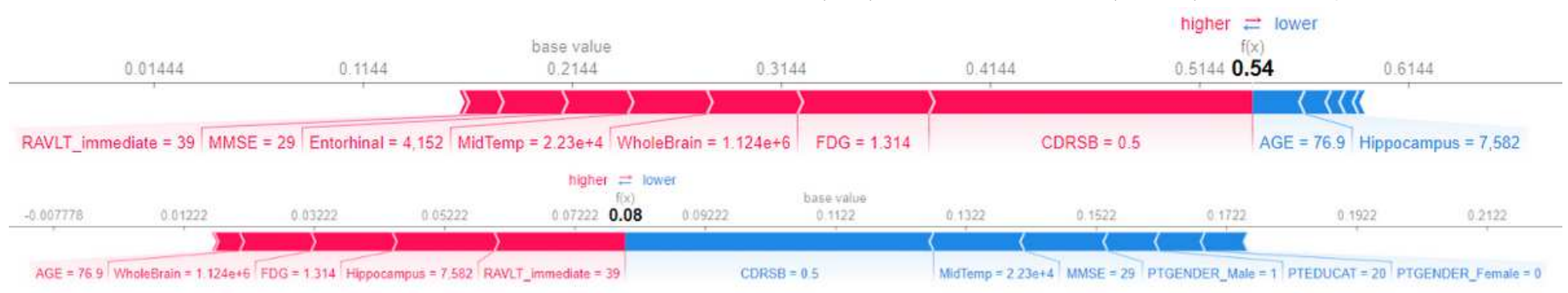

\section{Figure 24}

Comparison between features impact on the predicted (EMCl) and true class (SMC) of a subject.

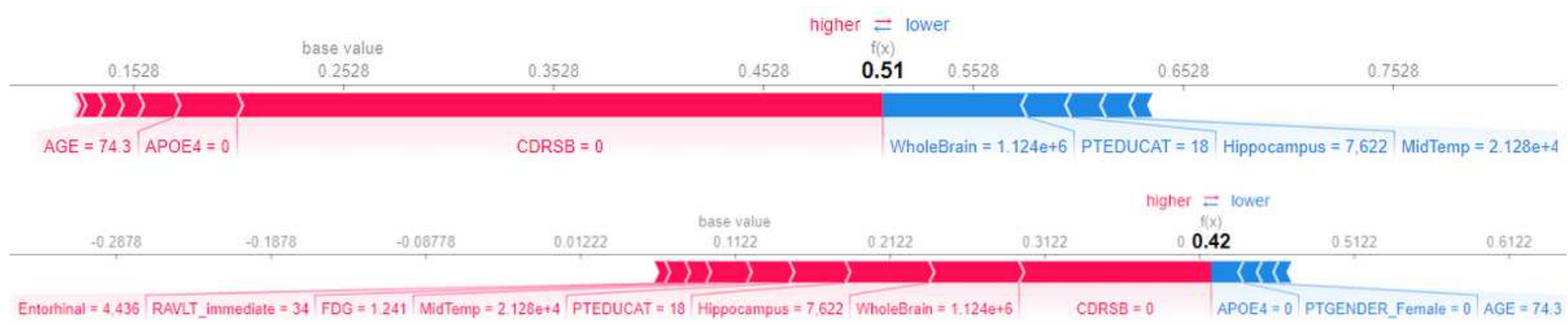

\section{Figure 25}

Comparison between features impact on the predicted (CN) and true class (SMC) of a subject. 


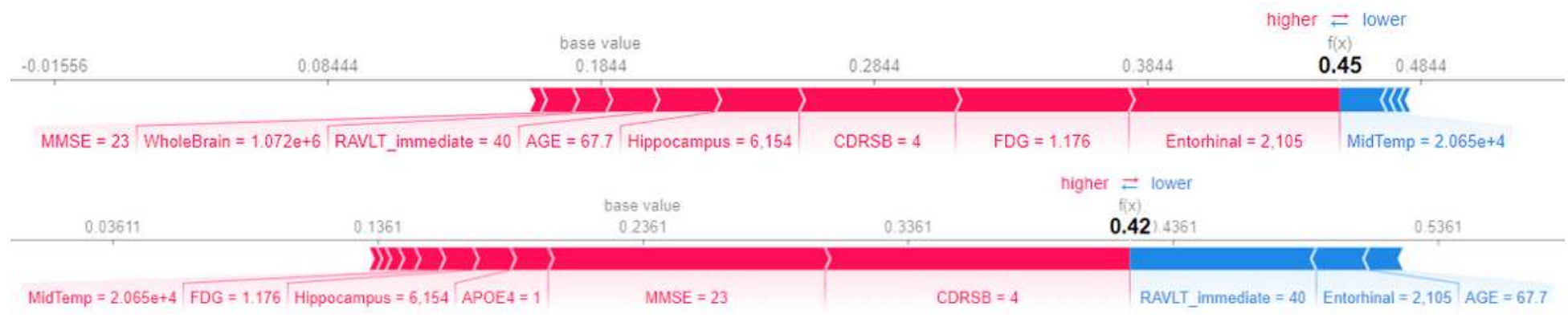

\section{Figure 26}

Comparison between features impact on the predicted ( $\mathrm{LMCl})$ and true class (AD) of a subject.

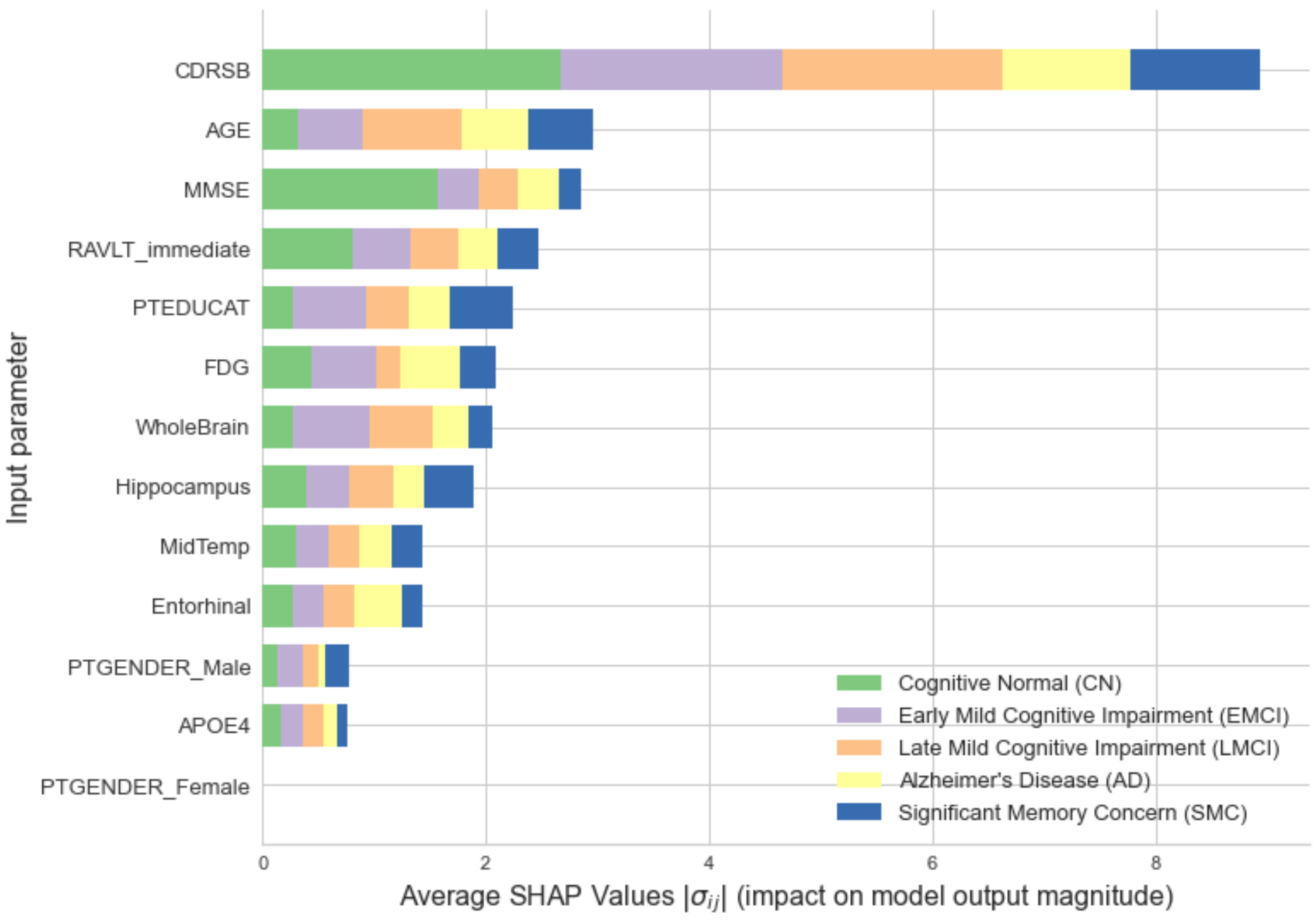

\section{Figure 27}

Summary variables importance plot for XGBoost model. 


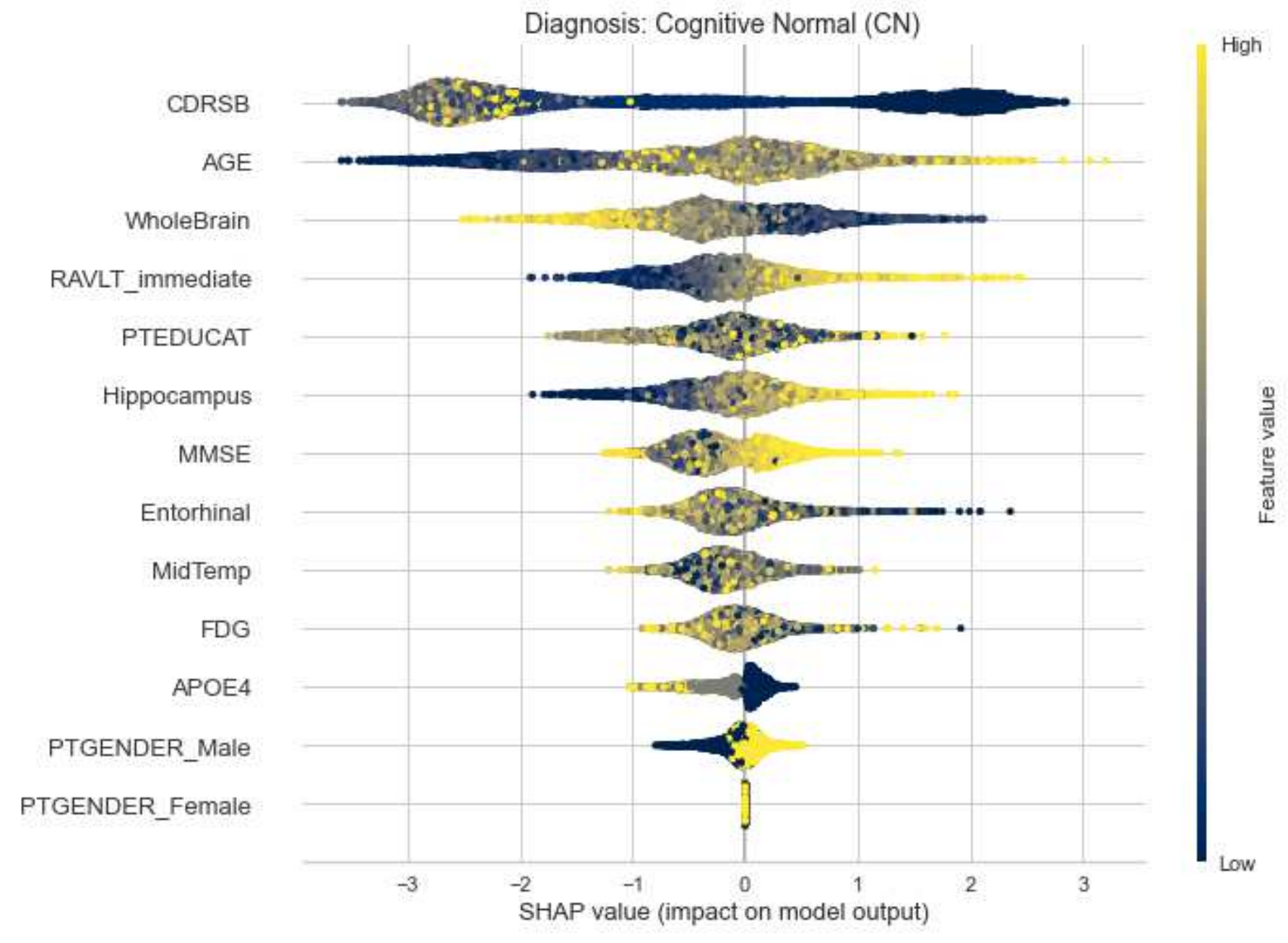

Figure 28

Variables importance plot for CN diagnosis. 


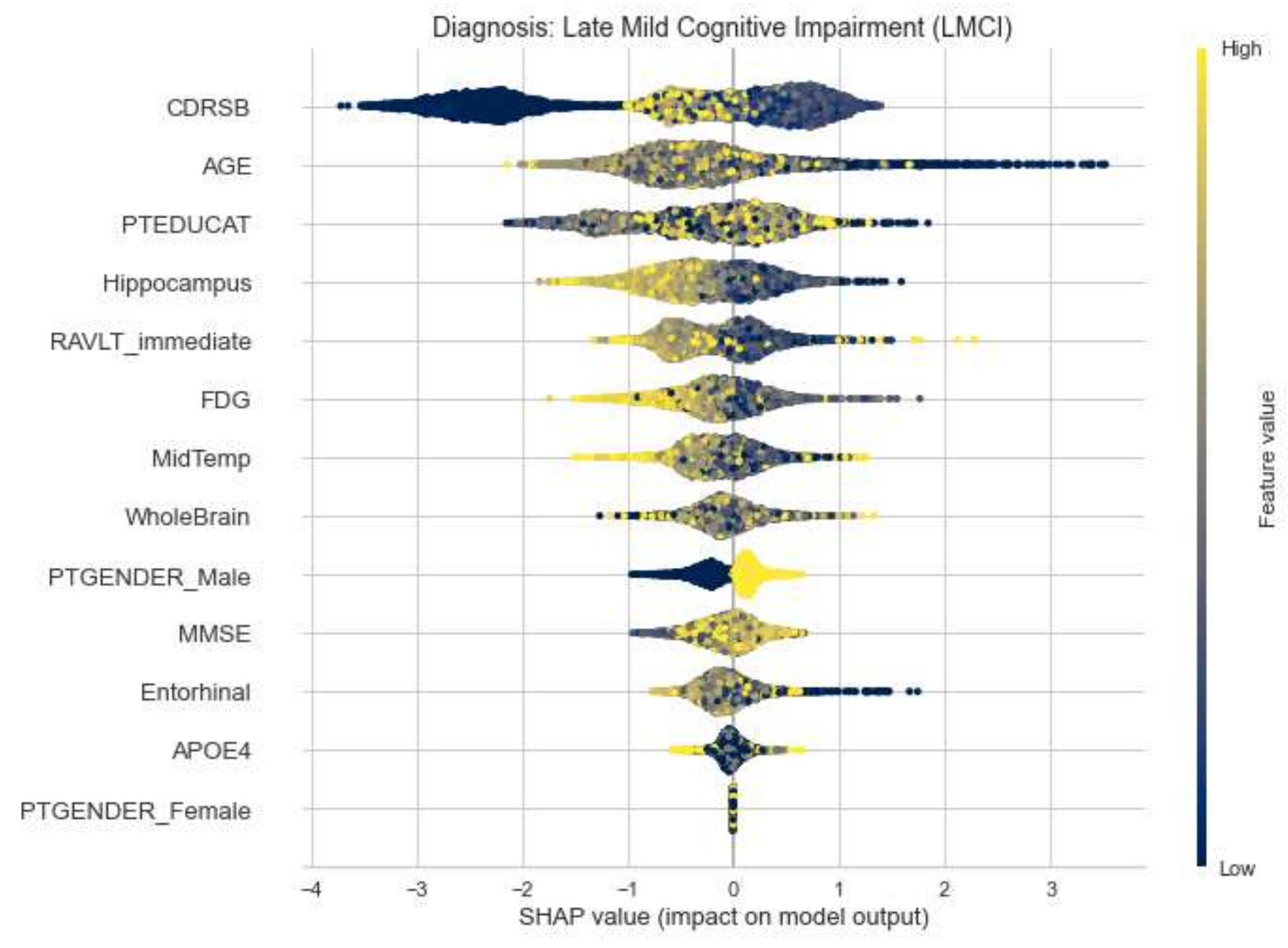

Figure 29

Variables importance plot for $\mathrm{LMCl}$ diagnosis. 


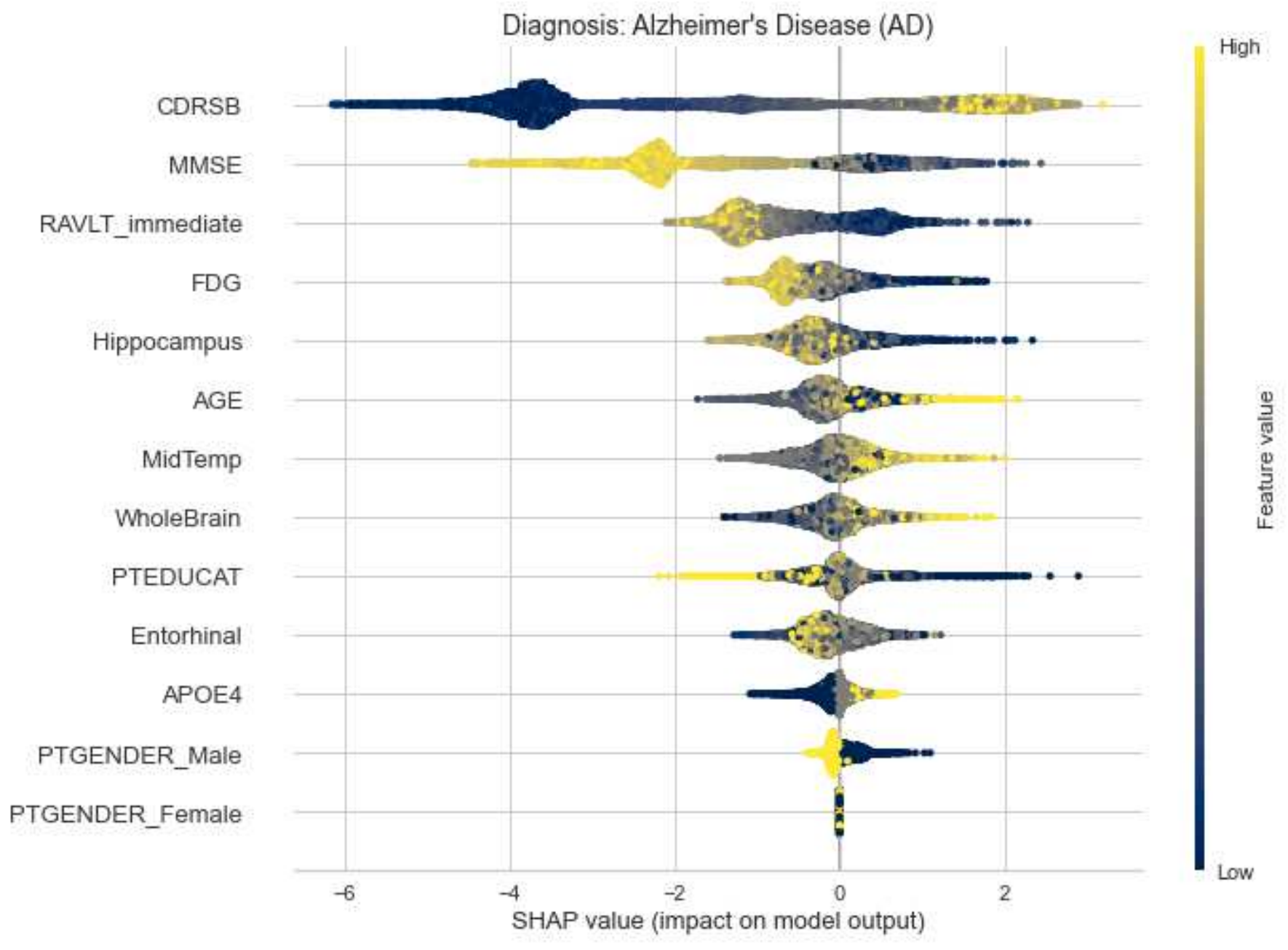

Figure 30

Variables importance plot for AD diagnosis 

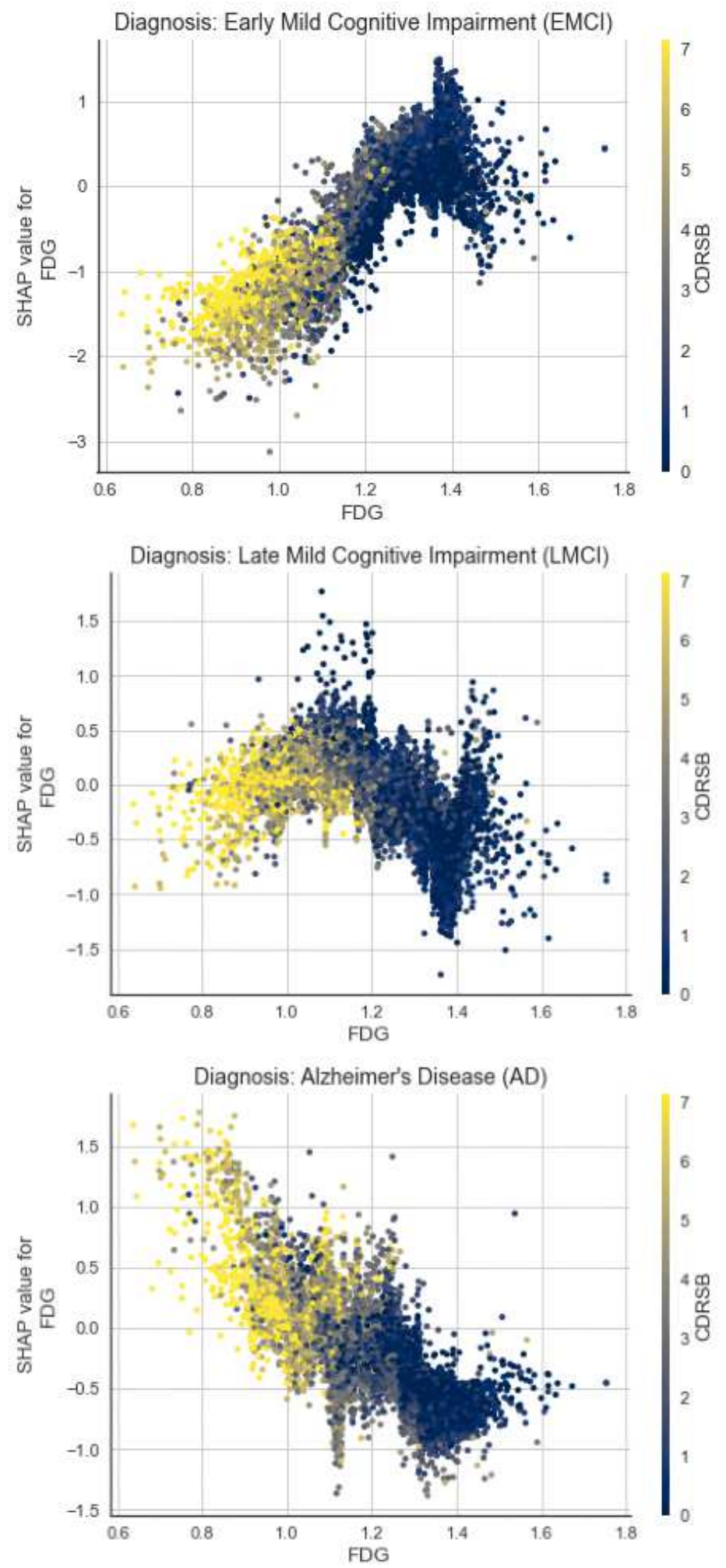

Figure 31

Dependence plots between FDG and CDRSB for different classes. 


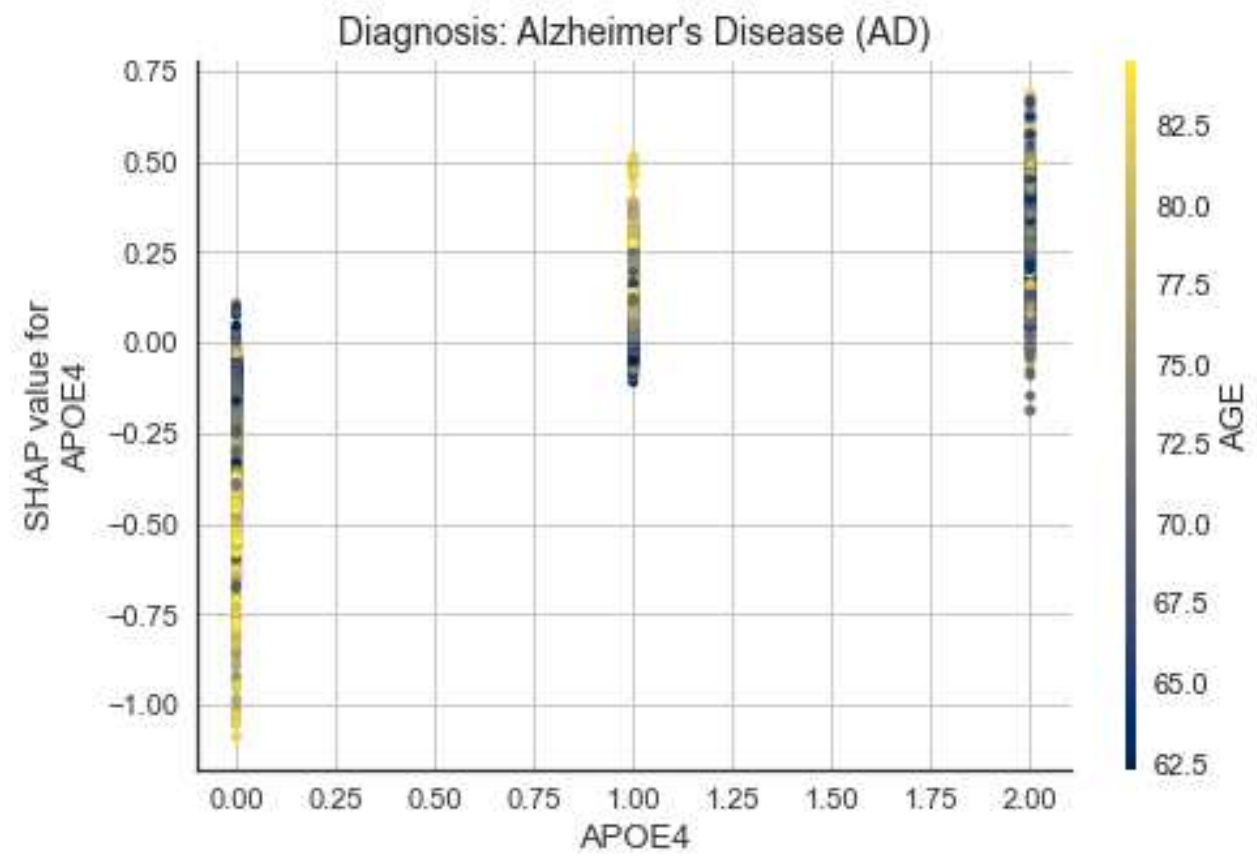

Figure 32

Dependence plot between APOE4 and AGE for AD class.

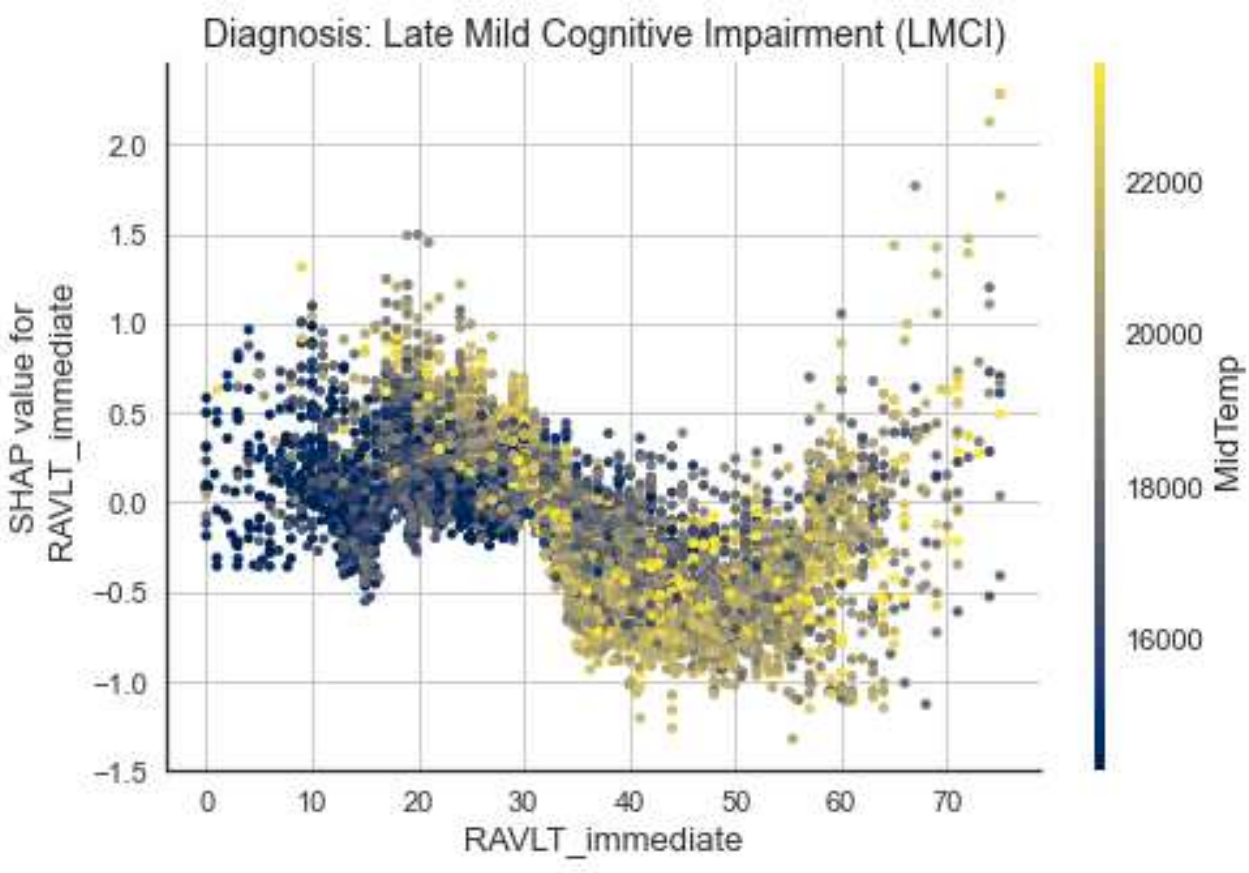

Figure 33

Dependence plot between RAVLT immediate and MidTemp for LMCl class. 


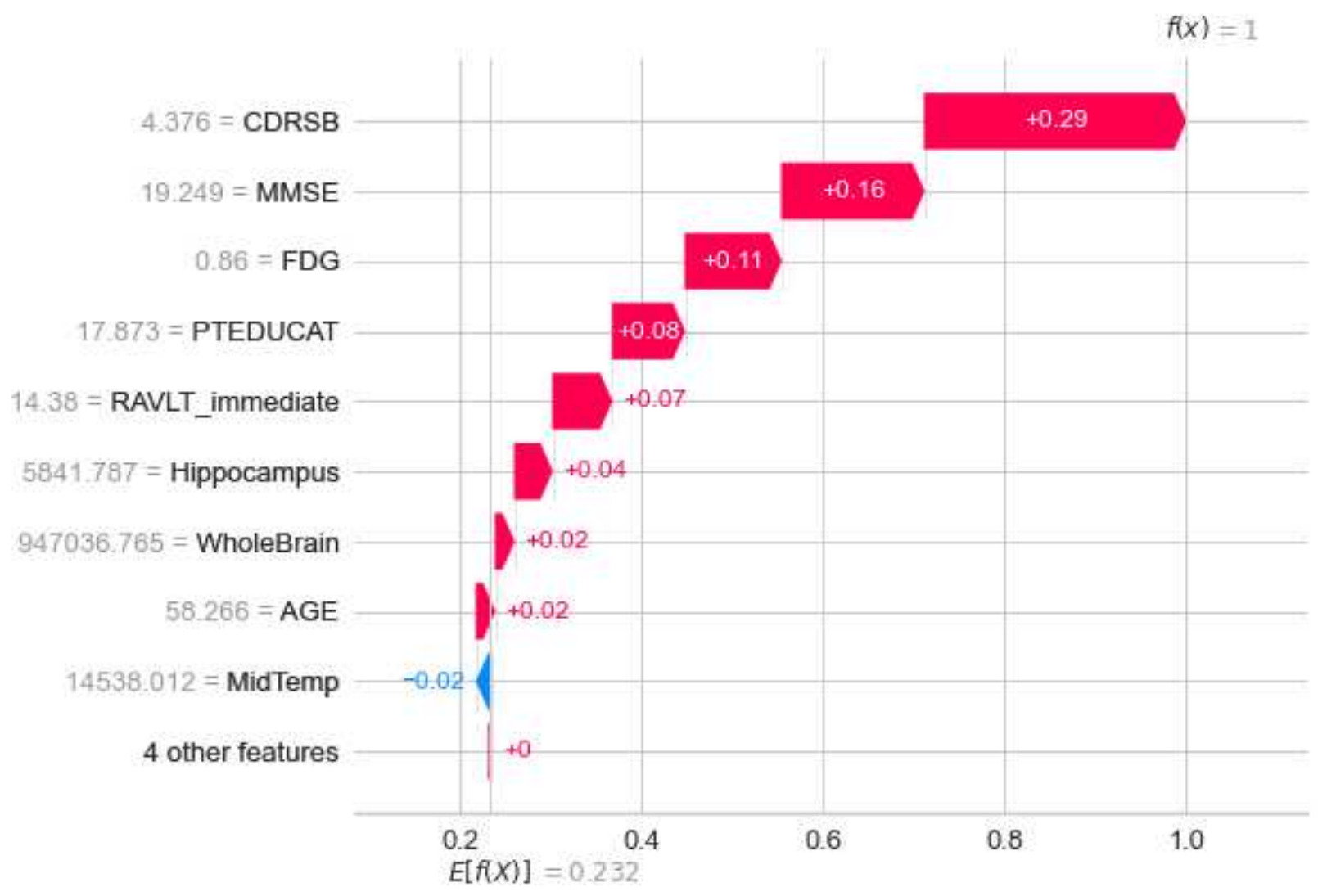

\section{Figure 34}

Features influence on a subject with $A D$ diagnosis to be predicted as $A D$.

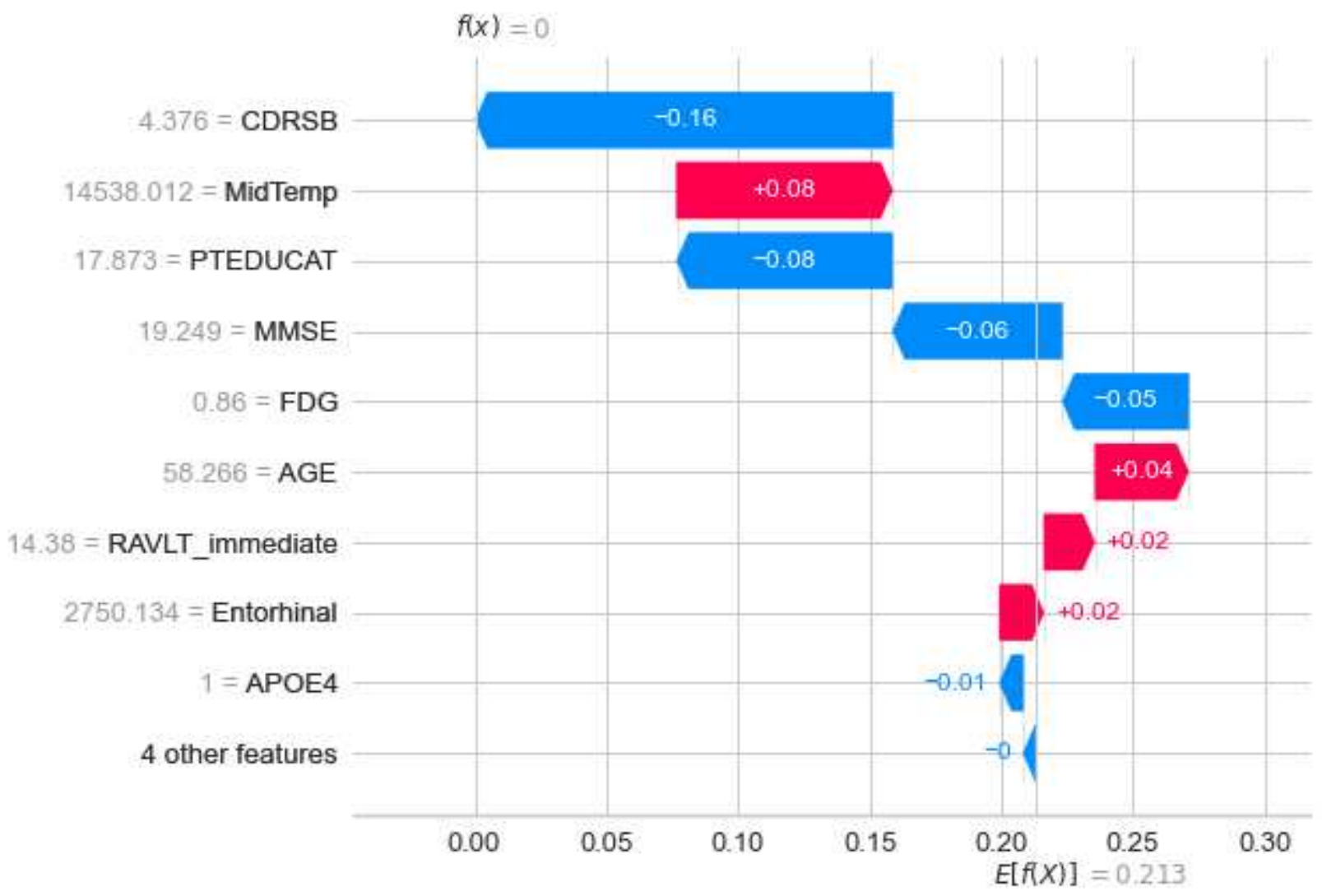




\section{Figure 35}

Features influence on a subject with $A D$ diagnosis to be predicted as $\mathrm{LMCl}$.

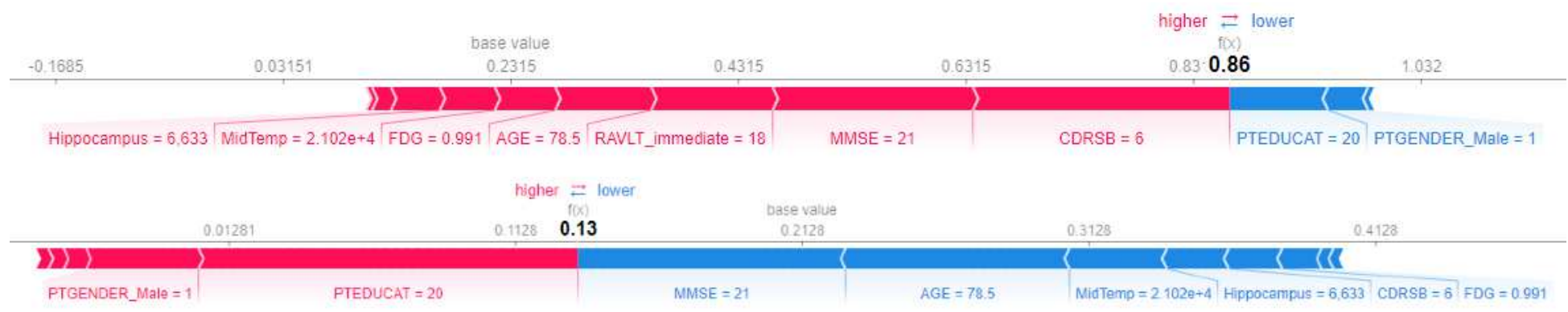

\section{Figure 36}

Comparison between features impact on the predicted (AD) and true class ( $L M C l)$ of a subject.

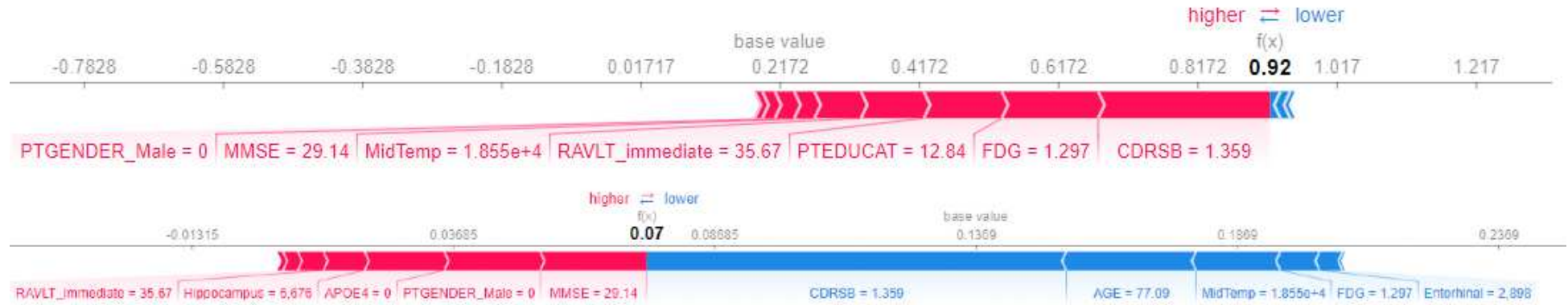

\section{Figure 37}

Comparison between features impact on the predicted (EMCl) and true class (SMC) of a subject.

0.001657
APOE $4=0$ AGE $=69.95$ RAVLT_immediate $=55.97$ MidTemp $=2.387 \mathrm{e}+4$

\section{Figure 38}

Comparison between features impact on the predicted (CN) and true class (SMC) of a subject

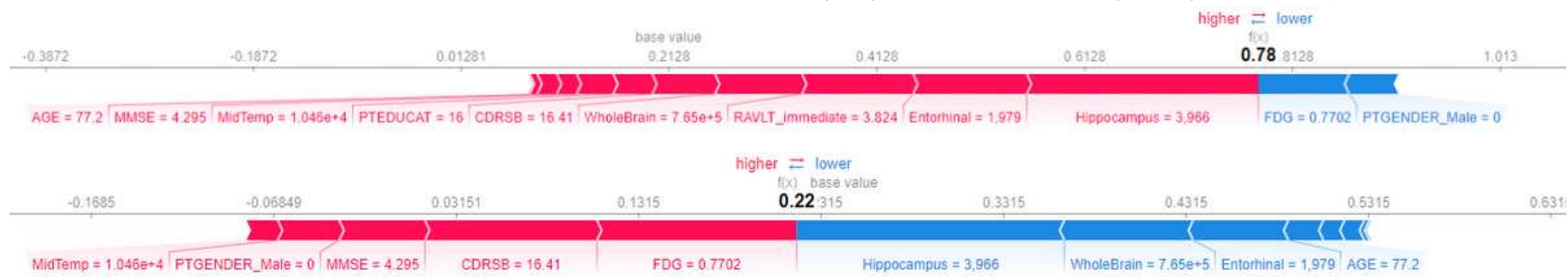

\section{Figure 39}


Comparison between features impact on the predicted (LMCI) and true class (AD) of a subject. 